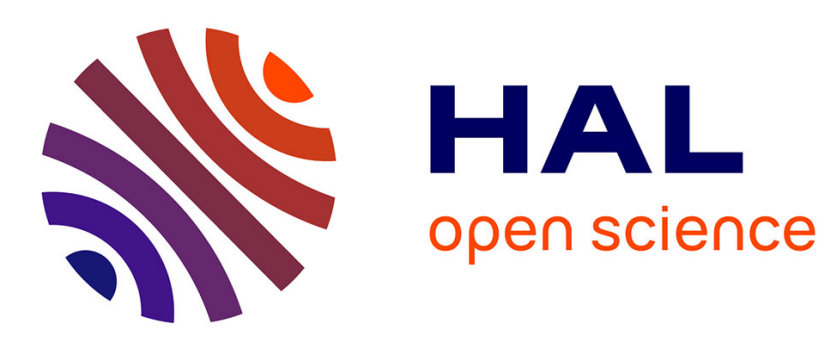

\title{
Inégalité de patrimoine et progressivité de l'impôt
}

Audrey Desbonnet, Jean-Olivier Hairault

\section{To cite this version:}

Audrey Desbonnet, Jean-Olivier Hairault. Inégalité de patrimoine et progressivité de l'impôt. Economie et Prévision, 2010, 2 (193), pp.21-41. hal-00519050

\section{HAL Id: hal-00519050 https://hal.science/hal-00519050}

Submitted on 21 Sep 2010

HAL is a multi-disciplinary open access archive for the deposit and dissemination of scientific research documents, whether they are published or not. The documents may come from teaching and research institutions in France or abroad, or from public or private research centers.
L'archive ouverte pluridisciplinaire HAL, est destinée au dépôt et à la diffusion de documents scientifiques de niveau recherche, publiés ou non, émanant des établissements d'enseignement et de recherche français ou étrangers, des laboratoires publics ou privés. 


\title{
Inégalité de patrimoine et progressivité de l’impôt
}

\author{
Audrey Desbonnet \\ Université de Vienne* \\ Jean-Olivier Hairault \\ PSE, Université Paris I Panthéon Sorbonne, IZA ${ }^{\dagger}$
}

Septembre 2009

\begin{abstract}
Résumé
On se propose d'analyser l'impact de la progressivité de l'impôt sur le revenu et sur les successions sur l'inégalité de patrimoine. Un modèle prenant en compte les principaux motifs d'accumulation permet de reproduire les inégalités de revenu et de patrimoine observées en France. En passant à une proportionnalité de chaque type d'impôt, on révèle que la progressivité de l'impôt sur le revenu permet de réduire davantage l'inégalité de patrimoine. La progressivité de l'impôt sur le revenu réduit le risque de revenu. L'épargne de précaution des plus riches se réduit et l'inégalité de patrimoine diminue. En revanche, la progressivité de l'impôt sur les successions modifie peu le risque de revenu. En outre, la progressivité de l'impôt sur les successions s'avère inefficace pour réduire l'inégalité de revenu à la différence de la progressivité de l'impôt sur le revenu.
\end{abstract}

Mots-clé : inégalité de patrimoine, progressivité de l'impôt, revenu, succession

Classification JEL : D31, D63, E62, H31

*Département d'économie (BWZ), Brünnerstrasse 72, A-1210 Vienne, Autriche. Tel. : [+43] 1-4277-37477, Fax : [+43] 1-4277-37498

${ }^{\dagger}$ Maison des Sciences Economiques, EUREQua, 106-112 Bd de l'Hôpital, 75647 Paris Cedex 13. E-mail :joh@univparis1.fr 


\section{Introduction}

En France, la distribution du patrimoine est fortement inégalitaire. L'analyse de Catherine Rougerie [2002], fondée sur l'examen des patrimoines pour trois vagues successives (1986, 1992 et 1998) révèle, qu'en 2000, les 10\% des ménages les plus riches détenaient $44 \%$ du patrimoine total, alors que les $50 \%$ des ménages les moins riches n'en possédaient que $9 \%$.

Pour réduire les inégalités de patrimoine, la progressivité de l'impôt doit-elle porter de façon continue au cours de la vie sur les flux de revenus du capital via l'impôt sur le revenu ou au contraire au moment de la transmission des stocks d'actifs par l'intermédiaire de l'impôt sur les successions? L'ambition de cet article est de fournir une analyse comparative de la progressivité des impôts sur le revenu et sur les successions telle qu'elle existe en France. Plus précisément, cette étude se propose d'apprécier la contribution de la progressivité de chacun de ces deux impôts à la réduction de l'inégalité de patrimoine. Pensée pour réduire les inégalités de revenu et donc de consommation, la progressivité de l'impôt sur le revenu a-t-elle limité l'inégalité de patrimoine? Si tel est le cas, dans quelle proportion la progressivité de l'impôt sur le revenu est-elle capable de limiter l'inégalité de patrimoine relativement à celle de l'impôt sur les successions? Il s'agit avant tout d'une analyse positive qui cherche à évaluer les effets des systèmes existants d'imposition du revenu et des successions en France. On ne cherche pas à tirer de conclusion générale sur l'optimalité de l'une par rapport à l'autre.

L'impôt progressif français sur les successions vise à amputer le stock de capital laissé par les héritiers à la différence de l'impôt progressif français sur le revenu qui a davantage vocation à freiner l'accumulation de capital. En France, la recette issue de l'impôt sur les successions est faible. Elle représente à peine 0,5\% du produit intérieur brut (Bozio [2007]) alors que la recette issue de l'impôt sur le revenu représente 3,3\% du PIB (Bourguignon et Bureau [1999]). Il semble que la ponction opérée par l'impôt progressif sur le revenu soit plus importante. En 1998, l'impôt sur le revenu comportait 7 tranches de revenu contre 8 pour l'impôt sur les successions. Si les taux marginaux supérieurs de l'impôt sur le revenu sont plus élevés ${ }^{1}$, ils s'appliquent à une distribution du revenu moins inégalitaire que celle des successions. Les 10\% des ménages dont le revenu est le plus élevé détenaient 32,5\% du revenu total en 1998. En revanche, les 10\% des successions les plus importantes représentaient 42,3\% des successions totales en 1994 (Arrondel et Laferrère[2001]). Davantage de ménages sont exempts de l'impôt sur les successions. En 1994, seulement $32 \%$ des successions donnaient lieu à paiement de droits de successions (Arrondel[2006]). Seuls 50\% des foyers imposables sont exonérés de l'impôt sur le revenu (Piketty [2001]). Ces premiers éléments de statistiques descriptives laissent entrevoir un barème de l'impôt sur le revenu plus progressif et l'impact respectif des impôts sur le revenu et sur les successions sur la distribution des revenus et des successions. Néanmoins, ils ne nous renseignent pas sur l'impact de la progressivité des impôts sur le revenu et

\footnotetext{
${ }^{1}$ Le taux marginal supérieur du barème de l'impôt sur le revenu valait 54\% en 1996 alors qu'à cette même date le taux marginal supérieur du barème de l'impôt sur les successions s'élevait à seulement $40 \%$.
} 
sur les successions sur la distribution du patrimoine. Les travaux sur ce thème ne manquent pas. Un certain nombre d'entre eux montrent que les legs sont importants pour comprendre l'inégalité de patrimoine (Davies [1982], Laitner [2001], De Nardi [2004], Gokhale, Kotlikoff, Sefton et Veale [2001]). Ces résultats suggèrent que l'impôt sur les successions pourrait permettre de réduire sensiblement l'inégalité de patrimoine en réduisant celle des successions. Castaneda, Diaz-Gimenez et Rios Rull [2003] montrent que la suppression de l'impôt progressif sur les successions aux Etats-Unis modifie à la hausse l'indice de Gini associé à la distribution du patrimoine, bien que de façon minime en raison de la faiblesse du taux d'imposition. En outre, Direr et Weitzenblum [2006] montrent qu'une suppression totale des droits de succession en France conduit à une augmentation significative de l'inégalité de patrimoine.

On peut se demander si la progressivité de l'impôt sur le revenu, dont l'objectif est de limiter les inégalités de revenu, a été en mesure de réduire également l'inégalité de patrimoine. L'analyse historique sur les hauts revenus français du $20^{\text {ème }}$ siècle conduite par Piketty [2001] souligne l'importance de la progressivité de l'impôt sur le revenu en matière de lutte contre l'inégalité de patrimoine. Elle permettrait de limiter les capacités d'accumulation du capital des plus fortunés, réduisant ainsi la concentration à venir des patrimoines et des revenus du capital. Castaneda, Diaz-Gimenez et Rios Rull [1998] montrent dans le cadre d'un modèle à agents hétérogènes que l'impôt progressif sur le revenu réduit davantage l'inégalité de patrimoine aux Etats-Unis que l'impôt proportionnel sur le revenu. Ventura [1999] évalue les conséquences distributionnelles, entre autres, d'une réforme fiscale visant à remplacer l'imposition américaine des revenus du capital actuelle par une imposition de type linéaire. Si la réforme est souhaitable du point de vue de l'accumulation du capital ${ }^{2}$, elle produit une distribution de salaire et de patrimoine plus concentrée.

Examiner des politiques économiques qui ont pour objet de réduire l'inégalité de patrimoine exige un diagnostic des mécanismes susceptibles de produire l'inégalité de patrimoine. Nombre d'économistes s'accordent pour dire que le patrimoine résulte de l'accumulation de capital et des transferts intergénérationnels. Parmi ces derniers on compte les donations entre vifs et les héritages qu'ils soient intentionnels ou non. Si aucun consensus n'émerge quant à la capacité de chaque source à expliquer le niveau et la forme de la distribution du patrimoine ${ }^{3}$, il n'en demeure pas moins que

\footnotetext{
${ }^{2}$ Il existe naturellement de nombreux travaux qui se focalisent sur le niveau de l'accumulation. Conesa et Krueger [2006] tentent de caractériser pour les Etats-Unis le barème optimal de l'imposition progressive du revenu. Ils aboutissent au résultat selon lequel l'impôt sur le revenu doit être moins pénalisant au bas et au haut de la distribution des revenus, la classe moyenne assumant la perte d'impôt générée par l'allègement des taux marginaux aux extrémités de la distribution des revenus. Domeij et Heathcote [2004] montrent que la réduction de l'imposition américaine des revenus du capital, supportée par un accroissement de l'imposition des revenus du travail, permet de stimuler l'investissement et d'accroître dans le long terme la consommation. Cependant, le coût à supporter à court terme d'une taxation des revenus du travail plus élevée est lourd pour tous les individus à l'exception des plus riches : ils doivent accepter une baisse de la consommation à court terme. Grant, Koulovatianos, Michaelides, et Padula [2003] montrent qu'une politique fiscale plus redistributive, aux Etats-Unis, conduit à une moindre inégalité de consommation en contrepartie d'une perte d'efficacité minime : l'épargne et la consommation moyenne diminuent.

${ }^{3}$ Kotlikoff et Summers [1981,1988] estiment la richesse de cycle de vie à environ $20 \%$ de la richesse nette américaine, alors que Modigliani [1988a,1988b] conclut que $80 \%$ ou plus de la richesse nette américaine peut être expliquée par l'épargne de cycle de vie.
} 
ces deux sources sont à l'origine de la distribution du patrimoine.

Le cadre théorique privilégié s'inscrit dans la lignée des travaux de Castaneda, Diaz-Gimenez et Rios Rull [1998, 2003]. Ils proposent une explication de l'inégalité de patrimoine aux Etats-Unis basée sur un modèle qui présente les caractéristiques du modèle dynastique et de cycle de vie. Du risque individuel de revenu et des contraintes d'endettement sont introduits. Dans ce cadre, ils montrent l'importance de prendre en compte l'héritage, l'altruisme intergénérationnel, mais également le système d'assurance retraite par répartition. Dans ce modèle, l'accumulation répond en partie à un motif d'épargne de précaution. Les titres sur les marchés financiers ne permettent pas de s'assurer parfaitement contre les risques individuels de revenu. Le risque individuel salarial est modélisé suivant un processus de Markov. Les individus sont supposés altruistes et par conséquent évaluent le montant de legs qu'ils désirent laisser à l'aune du bien être qu'il procure à leur descendance. Ainsi, les décisions d'épargne des individus intègrent les besoins d'épargne de leur progéniture.

Ce choix de modélisation permet d'une part de répliquer les caractéristiques importantes de l'économie française et d'autre part d'approcher les propriétés distributionnelles du revenu et du patrimoine. De ce point de vue, il offre un cadre théorique propice à l'analyse des effets, en matière d'inégalité, de la progressivité de l'impôt sur le revenu et sur les successions. Afin de juger de la contribution de la progressivité de chaque type d'imposition à la réduction de l'inégalité de patrimoine, leur mode de calcul est modifié. Le prélèvement des impôts sur le revenu et sur les successions devient proportionnel. L'étude est menée à poids constant de l'impôt.

On montre que la progressivité de l'impôt sur le revenu, telle qu'elle est instituée en France, permet de réduire davantage l'inégalité de patrimoine que la progressivité de l'impôt sur les successions. L'indice de Gini accuse une hausse plus marquée lorsque l'impôt sur le revenu devient proportionnel. Les implications divergentes en termes de comportement d'accumulation en sont la cause. Le passage à une imposition proportionnelle du revenu diminue ( resp. augmente) la facture d'impôt des agents riches (resp. pauvres) ce qui exacerbe le risque de revenu. En effet, la progressivité de l'impôt sur le revenu fournit une assurance contre le risque de revenu (Varian [1980], Eaton et Rosen [1980], Asdrubali, Sørensen et Yosha [1996]). Elle se substitue donc en partie à l'accumulation du capital qui est une forme d'auto-assurance contre le risque de revenu. Ce dernier se produit au cours de la vie active mais aussi également à la descendance des agents altruistes. Les agents épargnent en conséquence davantage pour le motif de précaution et d'autant plus qu'ils sont riches lorsque l'impôt sur le revenu devient proportionnel. Il s'ensuit une baisse du taux d'intérêt qui freine davantage l'accumulation de capital des agents pauvres. En revanche, le passage à une imposition de type proportionnelle des successions modifie peu l'ampleur du risque de revenu si bien que la hausse de l'épargne de précaution qui en découle est moindre. La progressivité de l'impôt sur les successions ne constitue donc pas une forme d'assurance suffisante contre le risque de revenu qui 
justifierait une diminution importante de l'épargne de précaution. Ainsi, la progressivité de l'impôt sur le revenu, telle qu'elle est introduite, en France, permet de réduire sensiblement l'accumulation des agents riches. En outre, la progressivité de l'impôt sur le revenu permet de diminuer la concentration du revenu (après impôt) et de la consommation de façon significative, à la différence de l'impôt sur les successions dont l'impact est négligeable. De ce point de vue, la progressivité de l'impôt sur le revenu s'avère relativement plus efficace en matière de réduction combinée des inégalités de patrimoine et de revenu.

La seconde section a pour objet de présenter le cadre théorique dans lequel l'analyse est conduite. La troisième section est consacrée à la présentation de l'étalonnage du modèle. La quatrième section offre une évaluation quantitative de la contribution de l'impôt progressif sur le revenu et sur les successions à à la réduction de l'inégalité de patrimoine. L'avant dernière section discute de la robustesse du résultat. La conclusion constitue la dernière section.

\section{L'environnement économique}

Le modèle considéré n'est autre que le modèle de croissance néo-classique stochastique pour lequel nombre d'hypothèses supplémentaires ont été faites. Un certain nombre d'entre elles sont centrales. Parmi elles, on compte le recours limité à l'emprunt, l'exposition à un risque individuel de salaire sur le marché de travail (dont on précisera par la suite la nature), une date de mort incertaine et l'incomplétude des marchés financiers. Ce sont autant d'hypothèses qui expliquent un comportement d'épargne dit de précaution (Aiyagari [1994]) et qui aboutit à des comportements hétérogènes en matière d'épargne.

A l'image de Castañeda, Díaz-Giménez et Ríos-Rull [1998, 2003], le modèle présente certains aspects du modèle de cycle de vie (les ménages travaillent puis prennent leur retraite) et du modèle dynastique (de nouveaux ménages naissent une fois que leurs parents meurent).

\subsection{La démographie}

La population est constituée de $J$ générations imbriquées. A chaque période un continuum de nouveaux ménages naît, remplaçant ceux qui meurent. Les nouveaux ménages croissent au taux constant $n$. Chaque ménage connaît une période d'activité qui lui donne droit à un salaire dicté par le processus stochastique idiosyncrasique. A la retraite $\left(j>j_{R}\right)$, le ménage touche une pension indexée sur sa carrière salariale. La date de mort est aléatoire et concerne uniquement les retraités. Soit $s$ la variable aléatoire qui indique conjointement l'âge du ménage et son revenu salarial. Les probabilités qui décrivent la transition d'une classe d'âge et de salaire à une autre classe d'âge et de salaire sont données par les probabilités conditionnelles définies ci dessous :

$$
\pi\left(s^{\prime} / s\right)=\operatorname{Pr}\left(s_{t+1}=s^{\prime} / s_{t}=s\right)
$$

Où $s$ et $s^{\prime} \in S$. 
On peut décomposer l'ensemble $S$ en deux sous ensembles $S_{1}$ et $S_{2}$ suivant le clivage actifs/retraités avec $S=S_{1} \cup S_{2}$.

\subsection{Dotations et Préférences}

\subsubsection{Le ménage}

Les ménages touchent durant leur vie active un salaire, contrepartie de leur participation au marché du travail. Le salaire peut faire l'objet de variations. Le salaire évolue en raison de l'effet dit d'expérience acquis avec l'ancienneté. Par ailleurs, il peut augmenter en raison du progrès technique qui profite à toute l'économie. Enfin, la diversité des carrières salariales trouve son origine, en partie, dans les fluctuations que peut connaître le salaire individuel. A chaque période de leur vie active, les ménages font face à un risque idiosyncrasique de revenu salarial. Ce risque idiosyncrasique de revenu salarial ne concerne que les ménages actifs. La classe de salaire, à laquelle les retraités appartiennent, est celle qu'ils occupaient lors de leur dernière période d'activité et qu'ils garderont durant toute la retraite.

Soit $\varepsilon \in E$ la variable aléatoire qui indique la classe de salaire du ménage. Les probabilités de transition qui décrivent l'évolution de la classe de salaire, sont données par les probabilités conditionnelles suivantes :

$$
\pi_{\varepsilon}\left(\varepsilon^{\prime} / \varepsilon\right)=\operatorname{Pr}\left(\varepsilon_{t+1}=\varepsilon^{\prime} / \varepsilon_{t}=\varepsilon\right)
$$

Où $\varepsilon$ et $\varepsilon^{\prime} \in E=\{1,2, \ldots, 10\}$

Les ménages tirent leur satisfaction du flux espéré de consommation, actualisé au taux $\beta \in] 0 ; 1[$, augmenté de l'utilité de leurs descendants. Ainsi, les préférences du ménage peuvent être décrites par la fonction d'utilité suivante :

$$
u\left(c_{0}\right)+\sum_{t=0}^{\infty} \beta^{t+1}\left\{\sum_{s_{t+1} \in \Omega} \pi\left(s_{t+1} / s_{t}\right) u\left(c_{t+1}\right)+\eta \beta \sum_{s_{t+1} \notin \Omega} \pi\left(s_{t+1} / s_{t}\right) V\left(a_{t+1}, s_{t+1}\right)\right\}
$$

où le flux instantané d'utilité $u$ est une fonction continue, strictement concave et qui satisfait les conditions d'Inada. On suppose en outre que la fonction d'utilité est de type CRRA :

$$
u(c)=\frac{c^{1-\sigma}}{1-\sigma}
$$

où $\sigma>1$ est le coefficient d'aversion relative pour le risque.

L'ensemble $\Omega$ désigne toutes les réalisations possibles de la variable aléatoire $s$ pour un ménage qui reste en vie ${ }^{4}$. Le second terme de l'équation décrit l'utilité retirée du bien être de sa descendance. Le paramètre $\eta$ indique l'ampleur de l'altruisme des ménages. Il est d'autant plus fort que ce paramètre est proche de 1 . Le legs sera d'autant plus conséquent que le ménage est altruiste. Pour $\eta=0$, le legs est accidentel. La fonction $V\left(a_{t+1}, s_{t+1}\right)$ désigne le bien être des descendants qui héritent du patrimoine $a_{t+1}$ et tirent un choc $s_{t+1}$. On suppose, en outre, que l'utilité espérée d'un ménage nouvellement né $V$ est la même qu'un ménage en âge de travailler.

\footnotetext{
${ }^{4}$ Il s'agit d'un sous ensemble de $S$ qui inclut $S_{1}$ et une partie de $S_{2}$.
} 


\subsubsection{L'entreprise}

La production de biens nécessite du capital $(K)$ et du travail $(L)$. La technologie de production, qui décrit le processus de production, est de type Cobb-Douglas et à rendements d'échelle constants. Elle vérifie par conséquent :

$$
Y_{t}=F\left(K_{t}, X_{t} L_{t}\right)=B K_{t}^{\alpha}\left(X_{t} L_{t}\right)^{1-\alpha}
$$

Le capital se déprécie au taux constant $\delta . B$ n'est autre qu'un paramètre d'échelle. $X_{t}$ désigne le progrès technique neutre au sens de Harrod. Il a pour effet d'améliorer le travail. On suppose que ce dernier croît au taux $\mu$ :

$$
X_{t+1}=(1+\mu) X_{t} \Longrightarrow X_{t}=(1+\mu)^{t} X_{0}
$$

Dans une économie où le progrès technique est neutre au sens de Harrod, les variables agrégées croissent au taux $\mu+n$, sur le sentier de croissance régulière. Les variables par tête croissent au rythme du progrès technique $\mu$. En revanche, le taux d'intérêt est constant le long du sentier de croissance équilibrée.

\subsection{Transmission de capital physique et humain}

Chaque fois qu'un ménage meurt, il est remplacé par un autre ménage qui hérite alors de son patrimoine. Ainsi, une partie de l'inégalité de patrimoine est transmise aux ménages nouvellement nés.

Les ménages naissent avec des niveaux de salaires différents, qui reflètent en partie la situation salariale des parents au sortir de la vie active. Ils sont donc susceptibles de faire des choix différents d'épargne qui concourent à l'inégalité de patrimoine. Si la situation salariale d'un ménage reflète l'accumulation de capital humain en partie hérité, l'inégalité de patrimoine trouve alors, en partie, son origine dans l'inégalité face à l'héritage de capital humain.

\subsection{Le gouvernement}

Le gouvernement a recours à deux types d'impôt pour redistribuer. L'un d'eux pénalise le revenu, l'autre réduit le montant des successions. Si le revenu du ménage est $y$, alors, le montant d'impôt sur le revenu dont le ménage doit s'acquitter est $t(y)$. Il est de type progressif et frappe les revenus du travail et du capital. L'impôt sur les successions est aussi progressif et intervient à l'entrée dans la vie active. Ainsi, si le ménage reçoit en héritage un patrimoine d'un montant égal à $a^{\prime}$, il doit au titre de l'imposition des successions $t_{h}\left(a^{\prime}\right)$. Le montant dont il hérite net de l'impôt sur les successions vaut $a^{\prime}-t_{h}\left(a^{\prime}\right)$.

\subsection{La caisse de retraite}

L'existence d'un système de retraite affecte les décisions en matière d'épargne à la veille de la retraite. Les ménages faiblement rémunérés ont besoin de peu de richesse ou d'aucune richesse, en 
présence d'un système de retraite. Leur consommation est largement financée par ce transfert de revenu. En revanche, pour des ménages ayant des revenus du travail élevés, la pension de retraite peut paraître insuffisante. Ils souhaitent alors davantage épargner (Gokhale, Kotlikoff, Sefton et Weale [2001] et Domeij et Klein [2002]). C'est pourquoi, il convient de modéliser le système de retraite dans la mesure où ce dernier participe à l'hétérogénéité de patrimoine de par les disparités de comportement d'épargne qu'il induit.

On suppose que les ménages durant la vie active cotisent auprès de la caisse de retraite qui, en retour, leur verse une pension durant leur retraite. Le montant dû au titre du financement de la retraite est proportionnel au revenu salarial. On note $\tau_{r}$ le taux proportionnel qui s'applique au revenu salarial. Ce dernier correspond au taux qui assure l'équilibre de la contrainte budgétaire de la caisse de retraite. Puisque les cotisations retraites sont proportionnelles au salaire, la pension versée, notée $p_{t}$, tient compte de l'effort de participation. On suppose que la pension de retraite est la somme pondérée du dernier salaire $w_{R-1}$ où $R$ désigne la date d'entrée en retraite et du salaire moyen des actifs $\bar{w}_{t}$ dans l'économie :

$$
p_{t}=\rho\left(\varrho \bar{w}_{t}+(1-\varrho) w_{R-1}\right)
$$

Cette modélisation calquée sur Hairault et Langot [2002] repose, d'une part, sur la volonté de reproduire certaines des caractéristiques du système de retraite français (que l'on précisera ultérieurement) et d'autre part sur la nécessité de prendre en considération le caractère redistributif de ce dernier. Lorsque $\varrho=0$, la pension de retraite correspond au dernier salaire. Dans ce cas,

on obtient une mesure du ratio de remplacement $\rho=\frac{p_{t}}{w_{R-1}}$. Lorsque $\varrho$ est non nul et positif, il rend compte du pouvoir redistributif du système de retraite. Plus $\varrho$ est faible et plus la pension de retraite est indexée sur le dernier salaire. L'inégalité de revenu et de patrimoine, qui découle en partie de l'hétérogénéité des trajectoires salariales, se répercute tout ou partie à l'entrée en retraite. Ainsi, un coefficient $\varrho$ élevé permet d'atténuer les inégalités de revenu et de patrimoine, au sortir de la vie active.

\subsection{Incomplétudes des marchés}

On suppose que les travailleurs ne peuvent que partiellement s'assurer contre le risque individuel de revenu salarial via les marchés financiers. En effet, ces derniers sont incomplets : il n'existe pas autant d'actifs contingents que d'état de la nature. En outre, on suppose qu'il n'est pas possible d'emprunter sur les marchés financiers. Ainsi, les ménages averses au risque et désireux de lisser leur consommation ont alors recours à l'épargne de précaution. On suppose par ailleurs qu'il n'existe aucun marché d'annuités qui permette de contracter une assurance contre le risque individuel de mortalité.

\subsection{L'équilibre}

Dans ce paragraphe nous nous attachons à définir l'équilibre macroéconomique. 


\subsubsection{Règles de décision des ménages}

Le vecteur des variables d'état est le vecteur $(a, s)$ où $a$ représente le patrimoine de début de période et $s$ la réalisation spécifique au ménage des évènements idiosyncrasiques. Soit $A$ l'ensemble des valeurs admissibles du patrimoine ${ }^{5}$. Le programme que résout le ménage, écrit sous sa forme récursive, est le suivant :

$$
v(a, s)=\max _{c \geq 0, a^{\prime} \in A} u(c)+\beta\left(\sum_{s^{\prime} \in \Omega} \pi\left(s^{\prime} / s\right) v\left(a^{\prime}, s^{\prime}\right)+\eta \sum_{s^{\prime} \notin \Omega} \pi\left(s^{\prime} / s\right) V\left(a^{\prime}-t_{h}\left(a^{\prime}\right), s^{\prime}\right)\right)
$$

sous les contraintes :

$$
\begin{aligned}
& c+a^{\prime}=a(1+r)+\left(1-\tau_{r}\right) w \varepsilon_{s}-t\left(a r+\left(1-\tau_{r}\right) w \varepsilon_{s}\right) \quad s \in S_{1} \\
& c+a^{\prime}=a(1+r)+p_{s}-t\left(a r+p_{s}\right) \quad s \in S_{2} \\
& a^{\prime} \geq 0 \\
& c>0
\end{aligned}
$$

Le temps n'apparaît pas car l'étude porte sur l'état stationnaire. Afin d'éliminer les effets de la croissance de la population et du progrès technique, on considère les variables par unité de travail efficace. On pose :

$$
\widehat{a}=\frac{a}{X L} ; \widehat{c}=\frac{c}{X L} ; \widehat{w}=\frac{w}{X L} ; \widehat{p}=\frac{p}{X L}
$$

Le programme que résout le ménage devient alors ${ }^{6}$ :

$$
v(\widehat{a}, s)=\max _{\widehat{c} \geq 0, \widehat{a}^{\prime} \in A} u(\widehat{c})+\widetilde{\beta}\left(\sum_{s^{\prime} \in \Omega} \pi\left(s^{\prime} / s\right) v\left(\widehat{a}^{\prime}, s^{\prime}\right)+\eta \sum_{s^{\prime} \notin \Omega} \pi\left(s^{\prime} / s\right) V\left(\widehat{a}^{\prime}-t_{h}\left(\widehat{a}^{\prime}\right), s^{\prime}\right)\right)
$$

sous les contraintes :

$$
\begin{aligned}
& \widehat{c}+\widehat{a}^{\prime}(1+g)=\widehat{a}(1+r)+\left(1-\tau_{r}\right) \widehat{w} \varepsilon_{s}-t\left(\widehat{a} r+\left(1-\tau_{r}\right) \widehat{w} \varepsilon_{s}\right) \quad s \in S_{1} \\
& \widehat{c}+\widehat{a}^{\prime}(1+g)=\widehat{a}(1+r)+\widehat{p}_{s}-t\left(\widehat{a} r+\widehat{p}_{s}\right) \quad s \in S_{2} \\
& \widehat{a}^{\prime} \geq 0 \\
& \widehat{c}>0
\end{aligned}
$$

Les solutions du programme récursif sont des fonctions qui indiquent le choix optimal d'épargne $\widehat{a}^{\prime}=f(\widehat{a}, s)$ et de consommation $\widehat{c}=h(\widehat{a}, s)$ quelle que soit la classe d'âge et de salaire $s$ à laquelle le ménage appartient et le patrimoine de début de période $\widehat{a}$.

\subsubsection{Définition de l'équilibre}

Soit $\lambda(\widehat{a}, s)$ la distribution de probabilité définie sur l'espace $\Xi=\left[0 ; \widehat{a}_{\max }[\times S\right.$. Celle-ci indique la proportion de ménages dont le patrimoine vaut $\widehat{a}$ et dont les classes d'âge et de salaire d'appartenance sont résumées par la variable aléatoire $s$. Définir l'équilibre stationnaire pour cette économie

\footnotetext{
${ }^{5}$ Etant donné la contrainte de non endettement qui pèse sur les agents, celle-ci prend ses valeurs dans $\mathbb{R}^{+}$. ${ }^{6} \widetilde{\beta}=\beta(1+g)^{1-\sigma}$ avec $(1+g)=(1+\mu)(1+n)$
} 
consiste à déterminer l'ensemble des règles de décision $\widehat{c}=h(\widehat{a}, s)$ et $\widehat{a}^{\prime}=f(\widehat{a}, s)$, les fonctions valeurs $v(\widehat{a}, s)$ et $V(\widehat{a}, s)$, la politique du gouvernement en matière de retraite et d'imposition du revenu et des successions $\left\{\tau_{r}, t(y), t_{h}\left(\widehat{a}^{\prime}\right)\right\}$, la distribution de probabilité $\lambda(\widehat{a}, s)$, le prix des facteurs $\{r, \widehat{w}\}$ et le stock de capital agrégé $\widehat{K}$ tels que :

(i) Les règles de décision $\widehat{c}=h(\widehat{a}, s)$ et $\widehat{a}^{\prime}=f(\widehat{a}, s)$ sont solutions du programme (12) sous les contraintes (13), (14), (15) et (16)

(ii) La distribution de probabilités associée à $\lambda(\widehat{a}, s)$ est une distribution stationnaire qui vérifie :

$$
\lambda\left(\widehat{a}^{\prime}, s^{\prime}\right)=\sum_{s} \sum_{\widehat{a}^{\prime}(\widehat{a}, s)} \pi\left(s^{\prime} / s\right) \lambda(\widehat{a}, s)
$$

$\lambda\left(\widehat{a}^{\prime}, s^{\prime}\right)$ s'interprète aussi comme le temps passé par le ménage dans cet état ${ }^{7}$.

(iii) Les prix des facteurs vérifient :

$$
\begin{aligned}
& r+\delta=F_{\widehat{K}}^{\prime}(\widehat{K}, 1) \\
& \widehat{w}=\frac{F_{L}^{\prime}(\widehat{K}, X L)}{X L}=F_{L}^{\prime}(\widehat{K}, 1)
\end{aligned}
$$

(iv) Le marché du capital est équilibré :

$$
\widehat{K}=\sum_{\widehat{a}} \sum_{s} \widehat{a}^{\prime}(\widehat{a}, s) \lambda(\widehat{a}, s)
$$

(v) La caisse de retraite est équilibrée :

$$
\tau_{r} / \sum_{\widehat{a}} \sum_{s \in S_{1}} \tau_{r} \widehat{w} \varepsilon_{s} \lambda(\widehat{a}, s)=\sum_{\widehat{a}} \sum_{s \in S_{2}} \widehat{p}_{s} \lambda(\widehat{a}, s)
$$

\section{Etalonnage du modèle}

La période du modèle est l'année. Le modèle est étalonné sur données françaises.

\subsection{La démographie}

On suppose qu'il existe 7 classes d'âge. Les quatre premières classes d'âge correspondent à la période d'activité. L'imposition des legs transmis a lieu durant la première classe d'âge. Les trois dernières classes d'âge caractérisent la retraite. Seuls les ménages de la dernière classe d'âge de la vie active sont concernés par le passage à la retraite. Une fois à la retraite, les ménages sont susceptibles de mourir. La probabilité de mourir est d'autant plus élevée qu'on est âgé.

Le ménage naît actif et hérite d'un montant positif ou nul de patrimoine amputé de l'impôt sur les successions (classe $C_{1}$ ). La vie active se poursuit (classe $C_{2}$ ). Puis, le ménage devient plus expérimenté sur le marché du travail, ce qui lui permet de bénéficier d'une amélioration de salaire (classe $C_{3}$ ). Sa vie active s'achève après une période de maturité qui lui donne droit à nouveau à

\footnotetext{
${ }^{7}$ En sachant qu'il y a autant d'états que de statuts possibles sur le marché du travail et de niveaux de richesse.
} 
une augmentation de salaire (classe $C_{4}$ ). Il débute alors sa période de retraite (Classe $C_{5}$ ) et reçoit une pension à ce titre sans que pèse le risque de mort. Une deuxième phase de retraite intervient durant laquelle la mort peut survenir (Classe $C_{6}$ ). Si le ménage vient à mourir, il est alors remplacé par $(1+n)$ agents qui appartiennent à la classe d'âge $C_{1}$. En revanche, si le ménage survit, il entre dans la dernière phase de sa vie (classe $C_{7}$ ) à l'issue de laquelle il meurt et est remplacé par $(1+n)$ agents. Les transitions d'une classe d'âge à une autre sont modélisées suivant un processus de Markov. Soit $\pi_{x}$ la matrice qui décrit les transitions d'une classe d'âge à une autre. Elle est de la forme :

$$
\pi_{x}=\left(\begin{array}{c|ccccccc} 
& C_{1} & C_{2} & C_{3} & C_{4} & C_{5} & C_{6} & C_{7} \\
\hline C_{1} & 0 & 1 & 0 & 0 & 0 & 0 & 0 \\
C_{2} & 0 & \pi_{22} & 1-\pi_{22} & 0 & 0 & 0 & 0 \\
C_{3} & 0 & 0 & \pi_{33} & 1-\pi_{33} & 0 & 0 & 0 \\
C_{4} & 0 & 0 & 0 & \pi_{44} & 1-\pi_{44} & 0 & 0 \\
C_{5} & 0 & 0 & 0 & 0 & \pi_{55} & 1-\pi_{55} & 0 \\
C_{6} & \pi_{61} & 0 & 0 & 0 & 0 & 1-\pi_{61}-\pi_{67} & \pi_{67} \\
C_{7} & \pi_{71} & 0 & 0 & 0 & 0 & 0 & 1-\pi_{71}
\end{array}\right)
$$

On suppose que la population croît au taux $n=0,65 \%$ par an (Charpin [1999]). Il reste à préciser le choix des valeurs de $\pi_{22}, \pi_{33}, \pi_{44}, \pi_{55}, \pi_{67}, \pi_{61}$ et $\pi_{71}$ de la matrice de transition $\pi_{x}$. On suppose que le temps moyen passé sur le marché du travail est de 40 ans (le nombre d'années nécessaires pour prendre sa retraite en France). Le temps moyen passé à la retraite est fixé à 20 ans. Le choix du temps moyen passé dans chaque classe d'âge active répond au souci de répliquer l'évolution moyenne des gains salariaux. Aussi, fixe-t-on la durée moyenne des classes d'âge $C_{2}, C_{3}$ et $C_{4}$ à 15 ans, 20 ans et 5 ans. On a par conséquent : $1-\pi_{22}=\frac{1}{15}, 1-\pi_{33}=\frac{1}{20}$ et $1-\pi_{44}=\frac{1}{5}$. Les différences de durée des périodes de retraite sont fixées afin d'approcher le ratio de dépendance entre actifs et retraités. Pour ce faire, on fixe à 4 ans le temps moyen durant lequel le retraité ne peut mourir (temps moyen passé dans la classe d'âge $C_{5}$ ). On a alors $1-\pi_{55}=\frac{1}{4}$. L'étalonnage des deux dernières phases de retraite (classes d'âge $C_{6}$ et $C_{7}$ ) intègre le fait que le ménage peut mourir et que la probabilité de mort croît avec l'âge. On fixe respectivement à 11 ans et 5 ans le temps moyen passé dans les classes d'âge $C_{6}$ et $C_{7}$. Il s'ensuit que : $\pi_{71}=\frac{1}{5}$. Puisque la probabilité de mort croît avec l'âge, $\pi_{61}$, la probabilité de mort lorsqu'on appartient à la classe d'âge $C_{6}$ doit être inférieure à $\pi_{71}$, la probabilité de mort lorsqu'on appartient à la classe d'âge $C_{7}$. On impose alors que $\pi_{67}=\frac{1}{11} \times \frac{6}{7}$ et $\pi_{61}=\frac{1}{11} \times \frac{1}{7}$.

\subsection{Préférences et taux d'escompte psychologique}

A l'image de Algan, Chéron, Hairault et Langot [2003], la valeur attribuée à l'aversion relative au risque est de 2,5. De façon générale, dans tous les modèles stochastiques à agents hétérogènes sa valeur est comprise entre 1 et 3 . Le taux de préférence pour le présent $\widehat{\beta}$ est fixé à 0,996 afin de reproduire un ratio $\frac{K}{Y}$ égal à 2, 4 (Hairault, Langot et Sopraseulth [2008]). 


\subsection{Risque de revenu et progression salariale}

On suppose que le risque de revenu salarial est identique quel que soit la classe d'âge du ménage actif. Ainsi, la matrice de mobilité salariale $\pi_{\varepsilon}$ est la même pour les classes d'âge $C_{1}, C_{2}, C_{3}$ et $C_{4}$. Son étalonnage est issu des estimations obtenues à partir du modèle DESTINIE (Colin [1999]). Cette dernière fournit la mobilité interdécile ${ }^{8}$ sur la période 1982-1992.

$\pi_{\varepsilon}=\left(\begin{array}{c|cccccccccc} & \mathrm{D} 1 & \mathrm{D} 2 & \mathrm{D} 3 & \mathrm{D} 4 & \mathrm{D} 5 & \mathrm{D} 6 & \mathrm{D} 7 & \mathrm{D} 8 & \mathrm{D} 9 & \mathrm{D} 10 \\ \hline \text { D1 } & 0,8735 & 0,0744 & 0,0208 & 0,0116 & 0,0033 & 0,0031 & 0,0039 & 0,0022 & 0,004 & 0,0031 \\ \text { D2 } & 0,0398 & 0,8261 & 0,0726 & 0,0378 & 0,0113 & 0,0026 & 0,0028 & 0,0037 & 0,0010 & 0,0024 \\ \text { D3 } & 0,0296 & 0,0620 & 0,7860 & 0,1017 & 0,0005 & 0,0085 & 0,0068 & 0,0012 & 0,0032 & 0,0006 \\ \text { D4 } & 0,0156 & 0,0128 & 0,0706 & 0,7593 & 0,1210 & 0,0053 & 0,0061 & 0,0060 & 0,0004 & 0,0029 \\ \text { D5 } & 0,0116 & 0,0108 & 0,0397 & 0,0842 & 0,7477 & 0,0864 & 0,0095 & 0,0018 & 0,0078 & 0,0005 \\ \text { D6 } & 0,0113 & 0,0039 & 0,0066 & 0,0002 & 0,1105 & 0,7575 & 0,0845 & 0,0177 & 0,0046 & 0,0031 \\ \text { D7 } & 0,0074 & 0,0060 & 0,0019 & 0,0026 & 0,0009 & 0,0958 & 0,7822 & 0,0857 & 0,0130 & 0,0046 \\ \text { D8 } & 0,0053 & 0,0015 & 0,0013 & 0,0021 & 0,0039 & 0,00347 & 0,0771 & 0,8021 & 0,0653 & 0,0068 \\ \text { D9 } & 0,0032 & 0,0021 & 0,0002 & 0,0004 & 0,0001 & 0,0047 & 0,0239 & 0,0659 & 0,8640 & 0,0354 \\ \text { D10 } & 0,0027 & 0,0005 & 0,0003 & 0,0001 & 0,0007 & 0,0015 & 0,0031 & 0,0137 & 0,0367 & 0,9406\end{array}\right)$

Elle indique la probabilité de passage d'un décile de salaire à un autre. Puisque le risque idiosyncrasique est apprécié au travers de la matrice de mobilité interdécile salariale, il s'ensuit que la différenciation des niveaux de salaire doit se faire aussi par décile. Pour ce faire, on a recours au panel DADS ${ }^{9}$ de 1994 (Piketty [1997]). Ce dernier indique le salaire moyen exprimé en francs par décile.

TAB. 1 - Salaire mensuel moyen par décile de salaire

\begin{tabular}{cccccccccc}
\hline \hline D1 & D2 & D3 & D4 & D5 & D6 & D7 & D8 & D9 & D10 \\
\hline 4820 & 5470 & 6180 & 6850 & 7540 & 8390 & 9390 & 10890 & 13770 & 23700 \\
\hline \hline
\end{tabular}

La grille de salaire vaut pour chaque classe d'âge avec, toutefois, une modification de celle-ci compte tenu de l'expérience acquise. Ainsi, l'échelle des salaires croît avec l'âge.

Les coefficients de progression salariale sont choisis de façon à reproduire l'évolution du salaire des actifs aux différents âges par rapport aux pensions des retraités durant le cycle de vie (Hairault et Langot [2008]).

\footnotetext{
${ }^{8}$ La matrice interdécile annuelle est obtenue en imposant : $\Pi_{1}=\Pi_{10}^{1 / 10}$ où $\Pi_{1}$ et $\Pi_{10}$ désignent respectivement la matrice de transition interdécile annuelle et décennale.

${ }^{9}$ Il s'agit des salaires mensuels, hors primes, nets de cotisations sociales et de CSG/RDS, concernent les salariés à plein temps du secteur privé
} 
TAв. 2 - Progression salariale le long du cycle de vie active

\begin{tabular}{cc}
\hline \hline C2 vers C3 & C3 vers $\mathrm{C} 4$ \\
\hline 1,30 & 1,39 \\
\hline \hline
\end{tabular}

\subsection{Altruisme et transmission de capital humain}

Les ménages, lorsqu'ils évaluent leur satisfaction, intègrent l'utilité que leur descendance peut tirer d'un éventuel legs. Le coefficient d'altruisme $\eta$ est fixé de façon à reproduire le poids des legs dans la richesse totale $\left(\frac{\operatorname{Legs}}{A}\right)$ de 1,4\% (Arrondel et Laferrère [1991]). Ceci nous conduit à fixer $\eta$ à 0,6 .

Il n'existe pas à notre connaissance de matrice de mobilité sociale interdécile. Toutefois, l'étude menée par Lefranc et Trannoy [2006] indique que l'élasticité du revenu des fils par rapport au revenu du père serait de 0,4 . Sur la base de cette information, nous construisons la matrice de mobilité sociale interdécile à l'image de De Nardi $[2004]^{10}$. Nous obtenons la matrice de mobilité sociale suivante :

$\left(\begin{array}{c|cccccccccc} & \text { D1 } & \text { D2 } & \text { D3 } & \text { D4 } & \text { D5 } & \text { D6 } & \text { D7 } & \text { D8 } & \text { D9 } & \text { D10 } \\ \hline \text { D1 } & 0,0548 & 0,1366 & 0,2508 & 0,2775 & 0,1851 & 0,0744 & 0,0180 & 0,0026 & 0,0002 & 0,0000 \\ \text { D2 } & 0,0293 & 0,0929 & 0,2090 & 0,2834 & 0,2313 & 0,1138 & 0,0337 & 0,0060 & 0,0006 & 0,0000 \\ \text { D3 } & 0,0145 & 0,0583 & 0,1606 & 0,2666 & 0,2666 & 0,1606 & 0,0583 & 0,0127 & 0,0017 & 0,0001 \\ \text { D4 } & 0,0067 & 0,0337 & 0,1138 & 0,2313 & 0,2833 & 0,2090 & 0,0929 & 0,0249 & 0,0040 & 0,0004 \\ \text { D5 } & 0,0029 & 0,0180 & 0,0744 & 0,1851 & 0,2775 & 0,2508 & 0,1366 & 0,0448 & 0,0088 & 0,0011 \\ \text { D6 } & 0,0011 & 0,0088 & 0,0448 & 0,1366 & 0,2508 & 0,2775 & 0,1851 & 0,0744 & 0,0180 & 0,0029 \\ \text { D7 } & 0,0004 & 0,0040 & 0,0249 & 0,0929 & 0,2090 & 0,2833 & 0,2313 & 0,1138 & 0,0337 & 0,0067 \\ \text { D8 } & 0,0001 & 0,0017 & 0,0127 & 0,0583 & 0,1606 & 0,2666 & 0,2666 & 0,1606 & 0,0583 & 0,0145 \\ \text { D9 } & 0,0000 & 0,0006 & 0,0060 & 0,0337 & 0,1138 & 0,2313 & 0,2834 & 0,2090 & 0,0929 & 0,0293 \\ \text { D10 } & 0,0000 & 0,0002 & 0,0026 & 0,0180 & 0,0744 & 0,1851 & 0,2775 & 0,2508 & 0,1366 & 0,0548\end{array}\right)$

\subsection{Le secteur productif}

Le taux de dépréciation et la part du capital dans le revenu sont respectivement fixés à 0,1 et 0,315. Le taux de progrès technique neutre au sens de Harrod est étalonné sur la base du taux de croissance de la productivité du travail : $\mu=2 \%$. Le paramètre d'échelle $B$ est fixé afin que le salaire soit égal à 1 . Le facteur travail est normalisé à 1 .

\footnotetext{
${ }^{10}$ De Nardi [2004] suppose que le niveau de productivité dont les ménages nouvellement nés héritent suit un processus $\operatorname{AR}(1)$ de persistence $\rho_{y h}$ et de variance $\sigma_{y h}$. Cette hypothèse permet alors de convertir le processus en chaîne de Markov, sur la base de la persistence et de la variance du processus selon la méthode de Tauchen et Hussey [1991]. Dans notre modèle, la persistence du processus AR(1) correspond à l'élasticité du revenu des fils par rapport au revenu du père égale à 0,4 . Puisque la classe de salaire, à laquelle les ménages appartiennent à leur naissance est donnée par la grille de salaire (Tableau 1), la variance du processus AR(1) est calculée à partir de cette grille de salaire.
} 


\subsection{La caisse de retraite}

L'étalonnage des paramètres $(\rho, \varrho)$ répond au souci de répliquer un certain nombre de faits stylisés qui concernent le statut des retraités relativement à celui des actifs. Afin d'approcher le ratio moyen des revenus issus du travail entre actifs et retraités, d'une valeur de 1,15 (Charpin [1999]), on fixe $\rho$ à 0,7436 . On pose $\varrho=0,054$ afin de répliquer le rapport interdécile des revenus hors patrimoine des retraités égal à 4,2 (Atkinson, Glaude, Olier et Piketty [2001]). On aboutit alors à un taux de cotisation de $30,51 \%$.

\subsection{Impôt progressif sur le revenu et sur les successions}

Dès lors que l'impôt est progressif, le taux marginal d'imposition cesse d'être constant ce qui rend son calcul moins aisé. Castanada, Diaz-Gimenez et Rios-Rull [1998, 2003] ont recours à une fonction estimée par Gouveia et Strauss $[1994]^{11}$ pour calculer l'impôt sur le revenu aux Etats-Unis. Cependant, nous ne disposons pas de l'équivalent sur données françaises. Le modèle ne prétend pas reproduire parfaitement les caractéristiques de la distribution du revenu et des successions telles que l'indice de Gini ou les fractiles. Autrement dit, le modèle ne reproduit pas parfaitement les niveaux relatifs et par conséquent les niveaux absolus de revenu et de succession. De fait, nos distributions du revenu et des successions ne sont pas à un changement d'échelle près la parfaite reproduction des distributions observées du revenu et des successions. Si cela avait été le cas, nous aurions pu appliquer aux seuils de tranche du barème de l'impôt sur le revenu le changement d'échelle. C'est pourquoi nous avons étalonné les seuils de tranche en les comparants aux déciles des distributions du revenu et des successions. En matière d'imposition des successions, il n'existe pas de fonction qui résume la forme du barème. Aussi, avons-nous eu recours à la même méthode pour étalonner les seuils de tranche du barème de l'impôt sur les successions.

\subsubsection{Impôt sur le revenu}

L'imposition des revenus est progressive. Le barème ${ }^{12}$ s'applique au revenu imposable pour une part (Quotient familial). Ce dernier se calcule en appliquant la déduction pour frais réel de $10 \%$ et l'abattement de $20 \%$ au revenu déclaré. Les taux utilisés dans le cadre du modèle sont reportés dans le Tableau 3. Il reste alors à étalonner les 6 seuils de tranche en identifiant leur place dans la distribution du revenu imposable pour une part. Le tableau 4 nous fournit la distribution du revenu déclaré du ménage par décile en francs ${ }^{13}$ (colonne 2 et 3 ).

\footnotetext{
${ }^{11}$ Celle-ci résume la forme du barème de l'impôt sur le revenu américain.

${ }^{12}$ source : Les hauts revenus en France au XXe siècle (p298), Piketty [2001]

${ }^{13}$ source : L'économie des inégalités, Piketty [1997]; Il s'agit des revenus annuels divisés par 12, comprenant les salaires, les revenus des indépendants, les retraites, les transferts et les revenus du patrimoine. Il s'agit, donc, de revenus nets de cotisations sociales et de CSG/RDS mais pas des autres impôts directs (IR, TH).
} 
TAB. 3 - Barème de l'impôt progressif sur le revenu en 1996

\begin{tabular}{cc}
\hline \hline Tranche de revenu imposable pour une part & taux \\
\hline moins de 25 610 Francs & $0 \%$ \\
de 25610 à 50 380 Francs & $10,5 \%$ \\
de 50380 à 88670 Francs & $24 \%$ \\
de 88670 à 143580 Francs & $33 \%$ \\
de 143580 à 233620 Francs & $43 \%$ \\
de 233620 à 288 100 Francs & $48 \%$ \\
plus de 288100 Francs & $54 \%$ \\
\hline \hline
\end{tabular}

On suppose que le ménage se compose d'un parent et de deux enfants ${ }^{14}$, à l'image de Ventura [1999]. Le barème de l'impôt progressif sur le revenu s'applique au revenu imposable pour une part (quotient familial). Le quotient familial s'obtient de la façon suivante :

$$
\text { quotient familial }=\frac{\text { revenu } \times 0.8 \times 0.9}{2}
$$

TAB. 4 - Distribution du revenu moyen en 1994

\begin{tabular}{|c|c|c|c|c|c|}
\hline Décile & Revenu moyen & Limite de décile & Revenu & Revenu imposable & Quotient familial \\
\hline D1 & 3070 & $\mathrm{P} 10$ & 4530 & 3262 & 19570 \\
\hline D2 & 5480 & P20 & 6425 & 4626 & 27756 \\
\hline D3 & 7290 & P30 & 8150 & 5868 & 35208 \\
\hline D4 & 9010 & $\mathrm{P} 40$ & 9880 & 7114 & 42682 \\
\hline D5 & 10740 & $\mathrm{P} 50$ & 11700 & 8424 & 50544 \\
\hline D6 & 12660 & $\mathrm{P} 60$ & 13730 & 9886 & 59314 \\
\hline D7 & 15000 & P70 & 16270 & 11714 & 70286 \\
\hline D8 & 17930 & P80 & 19790 & 14249 & 85493 \\
\hline D9 & 22390 & P90 & 25890 & 18641 & 111840 \\
\hline P90-95 & 28800 & P95 & 32040 & 23069 & 138410 \\
\hline P95-P100 & 47740 & P99 & 48336 & 34802 & 208810 \\
\hline & & P99,5 & 62945 & 45320 & 271920 \\
\hline & & $\mathrm{P} 99,9$ & 118205 & 85108 & 510650 \\
\hline
\end{tabular}

Note : -Les fractiles P99, P99,5 etP99,9 sont issus de Piketty [2001] (p 619)

-La dernière colonne correspond au quotient familial annualisé

Les données relatives à la distribution du revenu sont mensuelles. En revanche, les seuils de tranche du barème de l'impôt sur le revenu sont annuels. A des fins de comparaison, le quotient familial est annualisé (colonne 5 du tableau 4).

\footnotetext{
${ }^{14}$ Les situations familiales d'un foyer à l'autre diffèrent. Par conséquent, les factures d'impôt sont susceptibles de différer. Les enfants donnent lieu à des demi-part supplémentaires ce qui réduit de facto le revenu imposable par part auquel s'applique le barème de l'impôt sur le revenu. Toutefois, la modélisation adoptée ne permet pas de prendre en compte l'hétérogénéité des situations familiales. A des fins de simplicité, nous avons choisis de circonscrire le calcul de l'impôt sur le revenu au cas d'un ménage composé d'un parent et de deux enfants. A revenu imposable identique, la facture d'impôt d'un ménage sans enfant est sensiblement différente de celle d'un ménage avec deux enfants. Le risque de surestimer la facture d'impôt existe donc. En outre, les données de salaire et de mobilité salariale utilisées concernent l'individu. C'est pourquoi, nous avons considéré le cas d'un couple monoparental avec deux enfants.
} 
Si l'on note $t_{1}=25610 \mathrm{Frs}, t_{2}=50380 \mathrm{Frs}, t_{3}=88670 \mathrm{Frs}, t_{4}=143580 \mathrm{Frs}, t_{5}=233620$ Frs et $t_{6} .=288100 \mathrm{Frs}$, on obtient le classement suivant (position des seuils de tranche par rapport aux limites de décile du quotient familial annualisé) :

$$
\begin{aligned}
& P_{a} 10<t_{1}<P_{a} 20<P_{a} 30<P_{a} 40<t_{2}<P_{a} 50<P_{a} 60<P_{a} 70<P_{a} 80<t_{3} \\
& t_{3}<P_{a} 90<P_{a} 95<t_{4}<P_{a} 99<t_{5}<P_{a} 99.5<t_{6}<P_{a} 99.9
\end{aligned}
$$

Enfin, le système d'imposition des revenus est assorti d'un système de décote depuis la loi de Finances pour 1982. Celle-ci vise à alléger la charge fiscale des contribuables les plus modestes. La cotisation d'impôt est d'autant plus réduite que celle-ci est faible. Il constitue l'un des mécanismes qui est à l'origine de l'exonération d'un grand nombre de contribuables. En 1993, 3193000 de foyers étaient exempts de l'impôt progressif sur le revenu de par le mécanisme de la décote (Ducamin, Baconnier, Briet [1996]). Ainsi, tout agent, éligible au système de décote, c'est-à-dire dont la cotisation d'impôt est inférieure à la limite de décote bénéficie d'une réduction d'impôt. Cette mesure a pour effet d'accentuer le caractère progressif de l'impôt sur le revenu au bas de l'échelle des revenus. La remise est donnée par la formule arithmétique ci-dessous :

$$
t_{f}(y)=\left\{\begin{array}{c}
t(y) \text { si } t(y)>L D \\
t(y)-(L D-t(y)) \text { si } t(y) \leq L D
\end{array}\right.
$$

où $t(y), t_{f}(y)$ et $L D$ désignent respectivement l'impôt que doit acquitter l'agent, avant que le mécanisme de décote ${ }^{15}$ soit pris en compte, l'impôt final à payer et la limite de décote.

La limite de décote est fixée de façon à obtenir une valeur réaliste du nombre d'individus exempt de l'impôt progressif sur le revenu en 1994, égal à 50,1\% (Piketty [2001]).

\subsubsection{Impôt sur les successions}

A l'impôt progressif sur le revenu s'ajoute l'impôt sur les successions. Il porte sur le montant de legs laissé aux descendants. A la différence de l'impôt progressif sur le revenu, tout agent qui hérite y est soumis. Cependant, l'existence d'un abattement en ligne directe, exempte de celui-ci tous les héritiers qui reçoivent un legs inférieur à 300000 Francs. Les seuils d'imposition et les taux d'imposition ${ }^{16}$ qui s'appliquaient en 1996 sont ceux reportés dans le Tableau 5. On pose $t_{s 1}=300000 \mathrm{Frs}, t_{s 2}=350000 \mathrm{Frs}, t_{s 3}=375000 \mathrm{Frs}, t_{s 4}=400000 \mathrm{Frs}, t_{s 5}=3700000 \mathrm{Frs}$, $t_{s 6}=5900000 \mathrm{Frs}$ et $t_{s 7}=11500000 \mathrm{Frs}$ les seuils de tranche du barème de l'impôt progressif sur les successions. Le barème de l'impôt sur les successions ne s'applique pas au montant total légué mais à la part reçue par chacun des héritiers. Le legs soumis à l'imposition est donc divisé par deux puisque l'unité retenue est le ménage composé d'un parent et de deux enfants. La part nette que le ménage acquitte est ensuite multipliée par deux.

\footnotetext{
${ }^{15} \mathrm{La}$ formule de la décote est celle qui s'appliquait aux revenus de 1999 (source : www.assembleenationale.fr/budget/plf2000/g1861b11.asp).

${ }^{16}$ source : Les hauts revenus en France au XXe siècle (p770-771) Piketty [2001].
} 
TAB. 5 - Barème de l'impôt progressif sur les successions

\begin{tabular}{cc}
\hline \hline Tranche d'héritage imposable en ligne directe & taux \\
\hline moins de 300000 Francs & $0 \%$ \\
de 300000 à 350000 Francs & $5 \%$ \\
de 350000 à 375000 Francs & $10 \%$ \\
de 375000 à 400000 Francs & $15 \%$ \\
de 400000 à 3700000 Francs & $20 \%$ \\
de 3700000 à 5900000 Francs & $30 \%$ \\
de 5900000 à 11500000 Francs & $35 \%$ \\
plus de 11500000 Francs & $40 \%$ \\
\hline \hline
\end{tabular}

Les taux utilisés sont ceux décrits dans le Tableau 5. Les seuils de tranche sont déterminés selon la même méthode pour étalonner ceux de l'impôt progressif sur le revenu. Ils sont étalonnés de façon à reproduire leur classement par rapport au niveau moyen de chaque décile de la distribution des successions ${ }^{17}$.

TAB. 6 - Transmission moyenne par décile

\begin{tabular}{cccc}
\hline \hline D1 & 55330 & D7 & 512500 \\
D2 & 126500 & D8 & 669500 \\
D3 & 200250 & D9 & 984500 \\
D4 & 266500 & D10 & 2731000 \\
D5 & 333500 & P95 & 3925000 \\
D6 & 402000 & P99 & 8906500 \\
\hline \hline
\end{tabular}

On en déduit le classement suivant :

$$
\begin{gathered}
D 1<D 2<D 3<D 4<t_{S 1}<D 5<t_{S 2}<t_{S 3}<t_{s 4}<D 6 \\
D 6<D 7<D 8<D 9<D 10<t_{s 5}<P 95<t_{s 6}<P 99<t_{s 7}
\end{gathered}
$$

\subsection{Evaluation quantitative du modèle}

Il s'agit d'évaluer la capacité du modèle à reproduire certaines des caractéristiques de l'économie française.

\subsubsection{Les grandeurs macroéconomiques}

La part de la recette de l'impôt progressif sur le revenu dans le revenu imposable est surestimée puisque cette dernière valait 7, 4\% en 1996 (Bourguignon et Bureau [1999]). Le modèle surestime

\footnotetext{
${ }^{17}$ source : "Taxation and wealth transmission in France", p13 de Luc Arrondel et Anne Laferrère. Ces données sont elles même issues de l'enquête sur les mutations à titre gratuit de 1994 de la DGI
} 
quelque peu la part du montant de l'impôt progressif sur les successions dans le produit puisque celle-ci vaut $0,48 \%$ (Bozio [2007]).

TAB. 7 - Variables agrégées

\begin{tabular}{ccc}
\hline \hline & Données & Modèle \\
\hline$\frac{A}{Y}$ & 2,4 & 2,4 \\
$\frac{\text { legs }}{A}$ & $1,4 \%$ & $1,43 \%$ \\
$\frac{T_{I R}}{Y_{\text {imposable }}}$ & $7,4 \%$ & $8,9 \%$ \\
$\frac{T_{I S}}{Y}$ & $0,48 \%$ & $0,51 \%$ \\
$T_{I R}^{10 \%}$ & $63,3 \%$ & $60,5 \%$ \\
$T_{I S}^{10 \%}$ & $79,6 \%$ & $71,8 \%$ \\
Nombre d'individus exempt de l'IR & $50,1 \%$ & $50,7 \%$ \\
Nombre d'individus exempt de l'IS & $68,4 \%$ & $68,2 \%$ \\
\hline \hline
\end{tabular}

Note : -les symboles IR et IS renvoient respectivement à

l'impôt sur le revenu et sur les successions

- $T_{I R}^{10 \%}$ et $T_{I S}^{10 \%}$ renvoient respectivement au poids de l'impôt sur le revenu et sur les successions acquitté par les $10 \%$ des ménages les plus riches dans la recette totale

Le modèle sous-estime quelque peu le montant d'impôt sur le revenu acquitté par les $10 \%$ des ménages les plus riches en termes de revenu. En 1994, ils participaient à hauteur de 63,3\% de la recette issue de la collecte de l'impôt progressif sur le revenu (Piketty [2001]). Le modèle reproduit assez bien le nombre de ménages non soumis à l'impôt sur les successions (Arrondel et Laferrère [2001]). Enfin, le montant d'impôt sur les successions acquitté par les $10 \%$ des ménages les plus riches est sous-estimé (Arrondel et Laferrère [2001]).

\subsubsection{Les inégalités}

L'analyse de l'impact de la progressivité des impôts sur le revenu et sur les successions exige que le modèle soit en mesure de reproduire les principales propriétés de la distribution du revenu, du patrimoine et des transmissions successorales. Le modèle sous-estime quelque peu l'inégalité de revenu comme le suggère l'indice de Gini $^{18}$ et la proportion de revenu détenue par les $10 \%$ des ménages les plus riches en termes de revenu ${ }^{19}$. Le modèle ne parvient pas à reproduire parfaitement le haut de la distribution du patrimoine ${ }^{20}$, c'est-à-dire les $5 \%$ des ménages les plus riches.

\footnotetext{
${ }^{18}$ source : Chambaz, Guillaumat-Tailliet et Hourriez [1999]

${ }^{19}$ source : Piketty [2001].

${ }^{20}$ Les données relatives à la distribution du patrimoine français sont issues de Hourriez et Roux [2001], Kessler et Wolff [1991], Lollivier et Verger [1996] et Chambaz, Guillaumat-Tailliet et Hourriez [1999]. Lorsque la caractérisation de la distribution des richesses diffère quelque peu d'une étude à l'autre nous présentons les résulats sous forme d'intervalle.
} 
TAв. 8 - Inégalités de patrimoine, de revenu et de legs

\begin{tabular}{lcc}
\hline \hline & Données & Modèle \\
\hline Indice de Gini des revenus & $33 \%$ & $27,5 \%$ \\
revenu détenu par P95-P100 & $20,98 \%$ & $15,5 \%$ \\
revenu détenu par P90-P100 & $32,5 \%$ & $26,1 \%$ \\
Indice de Gini des salaires & $27 \%$ & $25 \%$ \\
Indice de Gini du patrimoine & {$[60 \% ; 70 \%]$} & $64,9 \%$ \\
Patrimoine détenu par P95-P100 & $51 \%$ & $29 \%$ \\
Patrimoine détenu par P90-P100 & {$[40 \% ; 50 \%]$} & $46,6 \%$ \\
Patrimoine détenu par P75-P100 & $70 \%$ & $74,3 \%$ \\
Patrimoine détenu par P0-P25 & $1 \%$ & $0,8 \%$ \\
Patrimoine détenu par P0-P50 & {$[7 \% ; 10 \%]$} & $7,4 \%$ \\
Legs détenu par P95-P100 & $30,4 \%$ & $36,2 \%$ \\
Legs détenu par P99-P100 & $13,4 \%$ & $9,4 \%$ \\
\hline \hline
\end{tabular}

L'absence d'entrepreneur dans le modèle en est la raison (Quadrini [2000], Cagetti et De Nardi [2006]). En outre, la concentration du patrimoine parmi les 10\% des ménages les plus riches en matière de patrimoine est assez bien appréhendée. Le modèle surestime la part du patrimoine détenu par le dernier quartile et reproduit assez bien la concentration au bas de la distribution du patrimoine. Le modèle sous-estime la concentration du patrimoine parmi les $5 \%$ des successions les plus importantes ${ }^{21}$.

\section{Evaluation quantitative de la progressivité des impôts sur le revenu et sur les successions}

L'objectif de cette section est d'apprécier la contribution de la progressivité actuelle de l'impôt sur le revenu et sur les successions à la réduction de l'inégalité de patrimoine. Le passage à un impôt proportionnel est mené à poids constant de l'impôt dans le revenu total, pour l'impôt sur les successions, et dans le revenu imposable pour l'impôt sur le revenu. Les taux proportionnels d'imposition du revenu et des successions s'élèvent respectivement à $8,9 \%$ et $14,7 \%$.

\subsection{Propriétés macroéconomiques}

Lorsque l'impôt sur les successions devient proportionnel, le patrimoine moyen accuse une très faible hausse. Cette dernière s'élève à $0,4 \%$. En revanche, lorsque l'impôt sur le revenu devient proportionnel, le patrimoine moyen augmente sensiblement, de 8,4\%. La hausse du patrimoine s'accompagne d'une baisse du taux d'intérêt quelque soit l'impôt. La baisse est plus remarquable lorsque l'impôt sur le revenu devient proportionnel (de l'ordre de 22,6\% contre 1,3\% dans le cas de l'impôt sur les successions).

\footnotetext{
${ }^{21}$ source : Taxation and Wealth transmission in France de Arrondel et Laferrère [2001].
} 
TAB. 9 - Variables agrégées

\begin{tabular}{cccc}
\hline \hline & Référence & IR proportionnel & IS proportionnel \\
\hline$A$ & 3,51 & 3,80 & 3,52 \\
$A$ & 2,40 & 2,54 & 2,41 \\
$\bar{Y}$ & $3,10 \%$ & $2,4 \%$ & $3,06 \%$ \\
$r$ & $3,45 \%$ & $3,8 \%$ & $3,48 \%$ \\
$\frac{\text { legs }}{Y}$ & $8,9 \%$ & $8,9 \%$ & $8,94 \%$ \\
$\frac{T_{I R}}{Y_{\text {imposable }}}$ & $0,51 \%$ & $0,62 \%$ & $0,52 \%$ \\
$\frac{T_{I S}}{Y}$ & $(60,5 \%, 0 \%)$ & $(24,7 \%, 5,3 \%)$ & $(60,4 \%, 0 \%)$ \\
$\left(T_{I R}^{10 \%, \text { riches }}, T_{I R}^{10 \%, \text { pauvres }}\right)$ & $71,8 \%$ & $75,7 \%$ & $59 \%$ \\
$T_{I S}^{10 \%}$ & $50,1 \%$ & $0 \%$ & $51,7 \%$ \\
Nombre d'individus exempt de l'IR & $68,2 \%$ & $70,6 \%$ & $29,2 \%$ \\
Nombre d'individus exempt de l'IS & $6 \%$ & \\
\hline \hline
\end{tabular}

Note : $T_{I R}^{10 \%, \text { riches }}$ désigne le poids de la facture d'impôt sur le revenu des $10 \%$ des ménages les plus riches dans la recette totale

Il apparaît que la progressivité de l'impôt sur le revenu influence significativement l'accumulation de capital privé, à la différence de celle de l'impôt sur les successions. L'accumulation de capital répond principalement à un motif d'assurance contre le risque de revenu. Le passage à une imposition de type proportionnelle du revenu réduit (resp. augmente) la facture d'impôt des ménages riches (resp. pauvres), ce qui exacerbe le risque de revenu. En effet, la facture d'impôt acquittée par les 10\% des ménages les plus riches ne représente plus que 24,7\% de la recette totale lorsque l'impôt sur le revenu devient proportionnel (Tableau 9). En revanche, la facture que doivent acquitter les $10 \%$ des ménages les plus pauvres cesse d'être nulle. Elle s'élève à 5, $3 \%$ de la recette totale de l'impôt sur le revenu. Ainsi, la progressivité de l'impôt sur le revenu réduit le niveau du risque de revenu et rend donc moins nécessaire l'auto-assurance. En revanche, la progressivité de l'impôt sur les successions ne réduit pas directement le risque de revenu ou peu. Elle diminue le rendement après impôt de l'accumulation pour un motif de legs.

TAB. 10 - Richesse moyenne le long du cycle de vie

\begin{tabular}{cccc}
\hline \hline & Référence & IR proportionnel & IS proportionnel \\
\hline$C_{2}$ & 2,41 & 2,54 & 2,44 \\
$C_{3}$ & 4,11 & 4,42 & 4,11 \\
$C_{4}$ & 4,73 & 5,16 & 4,74 \\
$C_{5}$ & 4,28 & 4,65 & 4,28 \\
$C_{6}$ & 3,38 & 3,74 & 3,39 \\
$C_{7}$ & 2,97 & 3,38 & 3 \\
\hline \hline
\end{tabular}


On retrouve cette différence d'effets quantitatifs le long du cycle de vie (Tableau 10). Lorsque l'impôt sur le revenu devient proportionnel, le patrimoine moyen s'accroît sensiblement quelle que soit la classe d'âge. En revanche, lorsque l'impôt sur les successions devient proportionnel, le patrimoine augmente peu. Bien que les retraités ne soient pas concernés par le risque de revenu ${ }^{22}$, leur patrimoine s'accroît lorsque l'impôt sur le revenu devient proportionnel. Le passage à une imposition proportionnelle du revenu exacerbe le risque de revenu. Les agents à la retraites souhaitent alors assurer leur descendance contre ce surcroît de risque. C'est la raison pour laquelle, le poids des legs dans le produit $\left(\frac{\text { legs }}{Y}\right)$ s'accroît lorsque l'impôt sur le revenu devient proportionnel. La progressivité de l'impôt sur les successions réduit (resp. accroît) le montant dont devront s'acquitter les héritiers pauvres (resp. riches) mais ne réduit pas ou très peu le risque de revenu des héritiers.

Cette analyse des effets de la progressivité de l'impôt sur les comportements d'accumulation révèle une différence fondamentale entre les deux types de progressivité. La progressivité de l'impôt sur le revenu joue directement sur l'importance des risques subis par les agents et par leurs descendants au cours de leur vie, tandis que la progressivité de l'impôt sur les successions influence davantage le prix de l'accumulation pour le motif de legs.

\subsection{Inégalités économiques}

Si l'objet de notre étude concerne principalement la distribution du patrimoine, les distributions de l'héritage, du revenu et de la consommation sont également examinées.

\subsubsection{Inégalité de patrimoine}

Le passage à une imposition proportionnelle, qu'elle porte sur le revenu ou sur les successions, provoque une hausse de l'indice de Gini. La hausse de l'indice de Gini est d'une ampleur différente. La hausse est plus prononcée lorsque l'impôt sur le revenu devient progressif ${ }^{23}$.

TAB. 11 - Indice de Gini du patrimoine

\begin{tabular}{ccc}
\hline \hline Référence & IR proportionnel & IS proportionnel \\
\hline $64,9 \%$ & $71,9 \%$ & $66,7 \%$ \\
\hline \hline
\end{tabular}

Lorsque les impôts sur le revenu et sur les successions deviennent proportionnels, le montant d'impôt que les agents pauvres (resp. riches) doivent acquitter augmente (resp. diminue). En outre,

\footnotetext{
${ }^{22}$ La pension que les retraités touchent est certaine.

${ }^{23}$ Notre étalonnage du ratio capital/PIB suppose que le patrimoine correspond au capital physique qui entre dans la fonction de production. Ainsi, le logement et les terrains ne sont pas pris en compte. Ce choix d'étalonnage peut conduire à sous-estimer le poids de l'imposition progressive des successions. Il faudrait étendre notre modélisation en équilibre général à tout le patrimoine. Toutefois, nous pensons que notre étude capte l'essentiel des mécanismes opposant impôt sur le revenu et impôt sur les successions.
} 
la proportionnalité de l'impôt sur le revenu, en modifiant la facture de l'impôt sur le revenu, exacerbe le risque de revenu. Les agents souhaitent davantage épargner pour motif de précaution tout particulièrement les agents riches. En revanche, la proportionnalité de l'impôt sur les successions n'affecte pas directement le risque de revenu ou peu. Elle accroît le rendement après impôt de l'accumulation pour un motif de legs. C'est pourquoi le comportement d'accumulation des ménages riches se modifie dans une moindre mesure. C'est par ce mécanisme que la proportionnalité de l'impôt sur le revenu accroît davantage l'inégalité de patrimoine. Ces résultats, en outre, indiquent que l'imposition des successions a des effets non négligeables sur l'inégalité de patrimoine. En effet, l'indice de Gini du patrimoine s'accroît de 1,8 points lorsque l'impôt sur les successions devient proportionnel. Bien que ce résultat ne soit pas directement comparable à celui de Direr et Weitzenblum [2006], il corrobore leurs résultats ${ }^{24}$, à savoir, que l'imposition de l'héritage a des effets significatifs sur l'inégalité de patrimoine.

L'analyse conduite sur l'ensemble de la population souffre, cependant, du caractère synthétique de l'indice de Gini. Une analyse, fondée sur la courbe de Lorenz, permet un examen plus approfondi des effets de la progressivité de l'impôt sur le revenu et sur les successions sur l'inégalité de patrimoine.

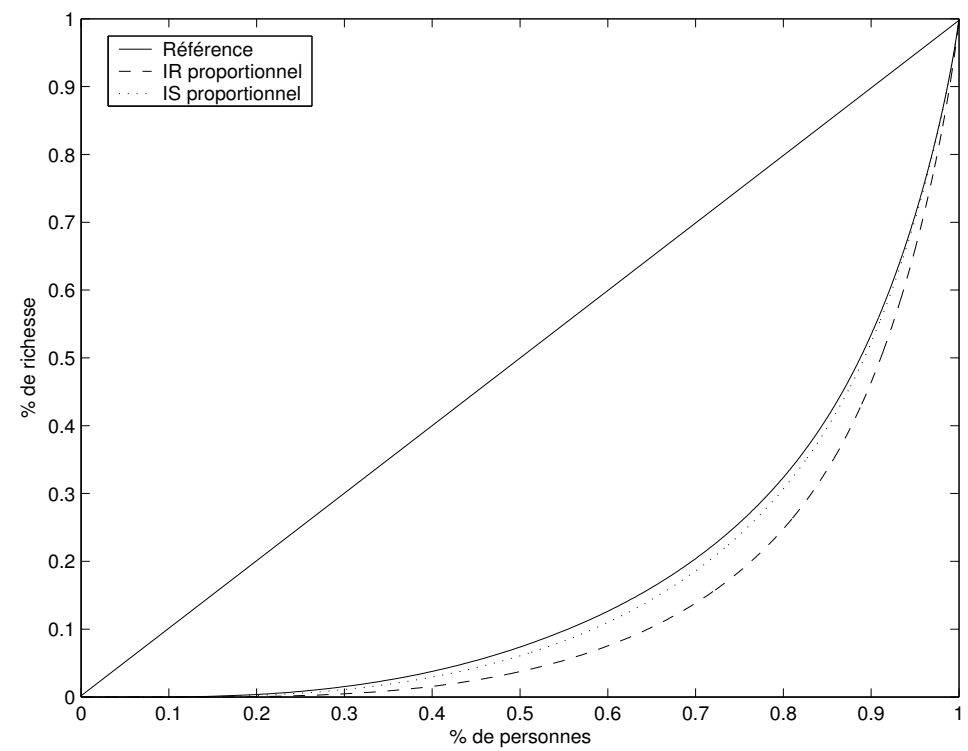

FIG. 1 - Courbe de Lorenz du patrimoine

Le passage à une imposition de type proportionnelle, quel que soit l'impôt, détériore principalement le sort des individus faisant parti des $70 \%$ des agents les plus pauvres (Graphique 1).

L'analyse de la concentration du patrimoine conforte les résultats issus de l'étude de l'indice de Gini et de la courbe de Lorenz. Réformer le mode de calcul de l'impôt sur le revenu et sur

\footnotetext{
${ }^{24}$ Direr et Weitzenblum [2006] montrent que la suppression des droits de successions conduit à un accroissement significatif de l'inégalité de patrimoine. L'indice de Gini passe de $67,2 \%$ à $70,4 \%$.
} 
les successions accroît sensiblement la concentration au haut de la distribution du patrimoine. Les agents les $10 \%$ les plus riches, suite au passage à une imposition du revenu de type proportionnelle, s'octroient dorénavant 7 points supplémentaire de patrimoine. En revanche, la hausse est moins remarquable lorsque l'impôt sur les successions devient proportionnel. Elle s'élève à 1,1 points.

TAB. 12 - Concentration du patrimoine

\begin{tabular}{lccc}
\hline \hline & Référence & IR proportionnel & IS proportionnel \\
P90-P100 & $46,6 \%$ & $53,6 \%$ & $47,7 \%$ \\
P75-P100 & $74,3 \%$ & $81,5 \%$ & $76,1 \%$ \\
P0-P25 & $0,8 \%$ & $0,19 \%$ & $0,56 \%$ \\
P0-P50 & $7,4 \%$ & $3,7 \%$ & $6,1 \%$ \\
\hline \hline
\end{tabular}

Le passage à une imposition de type proportionnelle quel soit que soit le type d'impôt considéré est fortement défavorable aux individus au bas de l'échelle patrimoniale. Les $25 \%$ des agents les plus pauvres ne détiennent plus que $0,2 \%$ du patrimoine lorsque l'impôt sur le revenu devient proportionnel. Lorsque l'impôt sur le revenu devient proportionnel, les agents pauvres exonérés de l'impôt sur le revenu, se voient contraints d'acquitter celui-ci. En outre, lorsque l'impôt sur le revenu devient proportionnel, le risque de revenu s'exacerbe. Les agents souhaitent davantage épargner pour le motif de précaution. L'offre de capital devient supérieure à la demande de capital. Le taux d'intérêt diminue (il passe de $3,1 \%$ à $2,40 \%$ ) afin de résorber l'excès d'offre de capital. Il devient plus coûteux de s'auto-assurer ce qui dissuade les agents pauvres d'épargner. En effet, le nombre d'agents contraints s'accroît fortement suite à la mise en place de l'impôt proportionnel sur le revenu (tableau 13).

TAB. 13 - Pourcentage d'agents contraints financièrement

\begin{tabular}{lc}
\hline \hline & Agents contraints \\
\hline Référence & $9,3 \%$ \\
IR proportionnel & $14,9 \%$ \\
IS proportionnel & $11 \%$ \\
\hline \hline
\end{tabular}

En revanche, la proportionnalité de l'impôt sur les successions s'avère moins pénalisante au bas de la distribution du patrimoine. Lorsque l'impôt sur les successions devient proportionnel, le montant d'impôt sur les successions dont devront s'acquitter les héritiers pauvres (resp. riches) augmente (resp. diminue) sans pour autant exacerber le risque de revenu des héritiers ou très peu. L'épargne de précaution des agents augmente peu et la baisse du taux d'intérêt qui s'ensuit est plus faible (le taux d'intérêt passe de $3,1 \%$ à $3,06 \%$ ). 
Il apparaît que la progressivité de l'impôt sur le revenu réduit davantage l'inégalité des successions. En effet, la hausse qu'accuse l'indice de Gini est plus marquée lorsque l'impôt sur le revenu devient proportionnel (Tableau 14).

\begin{tabular}{cccc} 
TAB. 14 - Propriétés distributionnelles des successions nettes de l'impôt \\
\hline \hline & Référence & IR proportionnel & IS proportionnel \\
\hline Indice de Gini & $72,8 \%$ & $78,5 \%$ & $76,5 \%$ \\
P75-P100 & $82 \%$ & $88,4 \%$ & $86,5 \%$ \\
P0-P50 & $3,1 \%$ & $0,8 \%$ & $1,40 \%$ \\
\hline \hline
\end{tabular}

Si la progressivité de l'impôt sur les successions permet d'accroître (resp. réduire) le montant d'impôt que doivent acquitter les héritiers riches (resp. pauvres), elle ne s'applique qu'une fois par génération et donc intervient à la fin du processus d'accumulation. En revanche, la progressivité de l'impôt sur le revenu s'applique à toutes les étapes du cycle de vie. En outre, la progressivité de l'impôt sur le revenu réduit l'importance du risque de revenu subi par les agents actifs et par leurs descendants. Les agents riches sont moins enclins à épargner. La progressivité de l'impôt sur le revenu freine leur accumulation et de facto réduit le patrimoine qui peut être accumulé et transmis à la mort.

En résumé, le passage à un impôt sur le revenu de type proportionnel diminue (resp. augmente) la facture d'impôt des agents riches (resp. pauvres). Le risque de revenu s'accroît et les agents épargnent davantage pour le motif de précaution et cela d'autant plus qu'ils sont riches. Il s'ensuit une baisse du taux d'intérêt qui freine d'autant plus l'accumulation de capital que l'agent est pauvre. Il en résulte un accroissement de l'inégalité de patrimoine. En revanche, le passage à une imposition proportionnelle des successions exacerbe peu le risque de revenu. L'accroissement de l'épargne de précaution des agents riches est en conséquence moindre. C'est la raison pour laquelle l'accroissement de l'inégalité de patrimoine est plus élevé lorsque l'impôt sur le revenu devient proportionnel.

\subsubsection{Inégalités de revenu}

Modifier la nature du prélèvement de l'impôt sur le revenu et sur les successions affecte très différemment l'inégalité de revenu. L'indice de Gini s'accroît sensiblement lorsque l'impôt sur le revenu devient proportionnel. En revanche, la hausse apparaît négligeable lorsque l'impôt sur les successions devient proportionnel. 
TAB. 15 - Propriétés distributionnelles du revenu net de l'impôt

\begin{tabular}{cccc}
\hline \hline & Référence & IR proportionnel & IS proportionnel \\
\hline Indice de Gini & $24 \%$ & $27,6 \%$ & $24,1 \%$ \\
\hline \hline
\end{tabular}

La déformation que la courbe de Lorenz subit (Graphique 2) est mineure lorsque l'impôt sur les successions devient proportionnel. En revanche, la proportionnalité de l'impôt sur le revenu conduit à une déformation plus marquée de la courbe de Lorenz. Cette dernière s'écarte davantage de la première bissectrice.

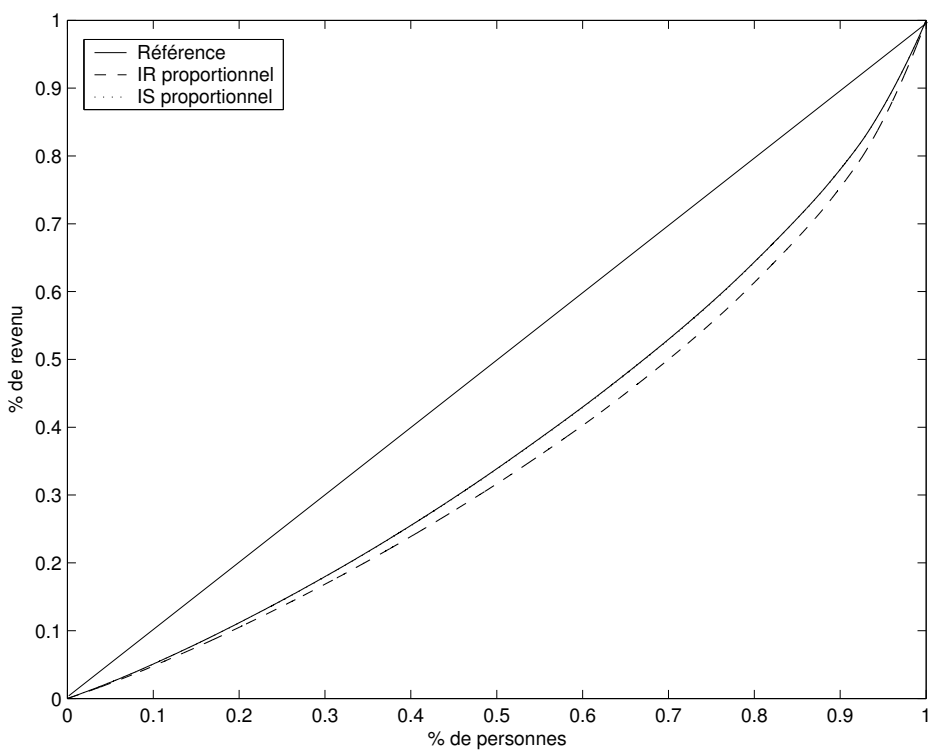

FIG. 2 - Courbe de Lorenz du revenu net de l'impôt

La progressivité de l'impôt sur le revenu diminue l'inégalité de revenu (net de l'impôt) en réduisant (resp. augmentant) la facture d'impôt des agents pauvres (resp. riches) mais également en réduisant l'épargne des agents riches. Bien que la progressivité de l'impôt sur les successions favorise les legs au bas de la distribution des successions, ces derniers sont trop petits pour augmenter sensiblement le revenu de ces agents pauvres. C'est pourquoi l'impôt sur les successions est inapproprié pour réduire l’inégalité de revenu.

\subsubsection{Inégalité de consommation}

Les implications différentes en termes d'inégalité de revenu se retrouvent dans l'inégalité de consommation. Le passage à un prélèvement proportionnel de l'impôt sur le revenu ou sur les successions affecte différemment la consommation suivant l'impôt considéré. 
TAB. 16 - Propriétés distributionnelles de la consommation

\begin{tabular}{cccc}
\hline \hline & Référence & IR proportionnel & IS proportionnel \\
\hline Indice de Gini & $16,6 \%$ & $18,8 \%$ & $16,6 \%$ \\
\hline \hline
\end{tabular}

L'indice de Gini s'accroît sensiblement lorsque l'impôt sur le revenu devient proportionnel. En revanche, celui-ci est inchangé lorsque l'impôt sur les successions devient proportionnel. L'analyse de la courbe de Lorenz de la consommation (Graphique 3) confirme les résultats issus de l'analyse de l'indice de Gini.

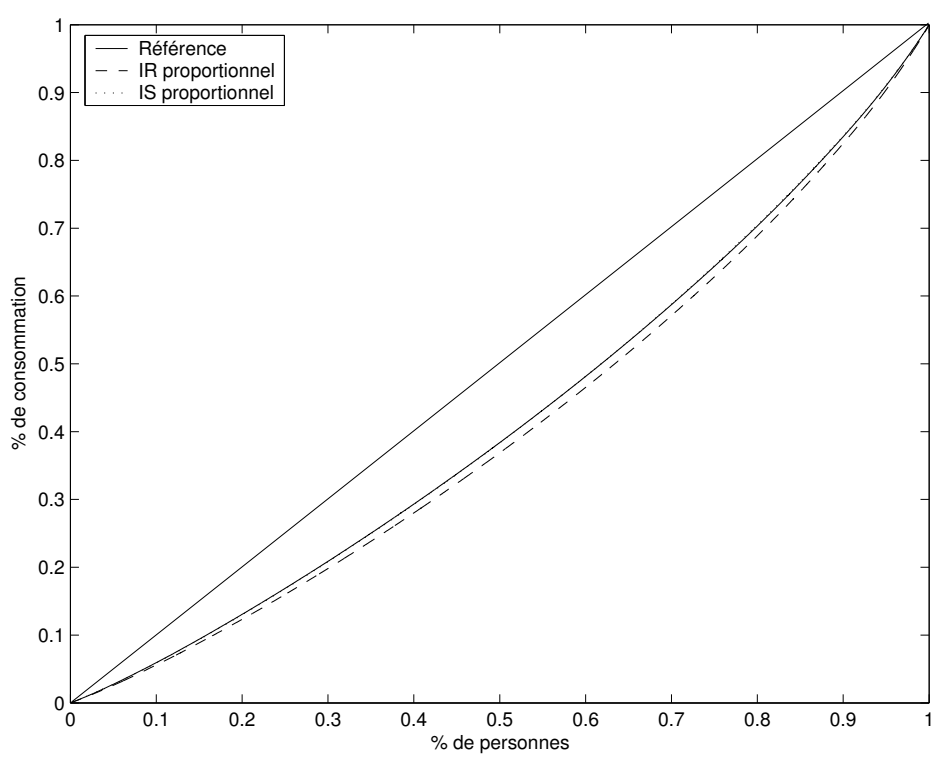

FIG. 3 - Courbe de Lorenz de la consommation

Lorsque l'impôt sur le revenu devient proportionnel, la courbe de Lorenz du revenu s'écarte davantage de la $1^{\text {ère }}$ bissectrice. En revanche, la courbe de Lorenz ne se modifie pas lorsque l'impôt sur les successions devient proportionnel. La proportionnalité de l'impôt sur le revenu réduit (resp. accroît) le revenu des agents pauvres (resp. riches) et de facto diminue (resp. augmente) la consommation des agents pauvres (riches). C'est la raison pour laquelle l'inégalité de consommation s'accroît. Ainsi, la progressivité de l'impôt sur le revenu semble jouer un rôle crucial dans la réduction des inégalités de consommation. Parce qu'elle a réduit l'importance du risque de revenu, elle a affecté à la baisse, significativement, le degré d'inégalité de la distribution de la consommation.

\section{Robustesse}

Cette section est consacrée à :

1. l'étude du rôle joué par le taux d'intérêt dans l'accroissement de l'inégalité de patrimoine lorsque les impôts progressifs sur le revenu et sur les successions deviennent proportionnels. 
Pour se faire, on se place dans le cadre d'une petite économie ouverte (le taux d'intérêt est invariant à tout déséquilibre apparaissant sur le marché du capital)

2. l'étude de l'impact de la proportionnalité de l'impôt sur le revenu sur l'inégalité de patrimoine en présence d'un mécanisme de décote.

\subsection{Analyse en équilibre partiel}

Lorsque l'impôt sur le revenu devient proportionnel, la facture d'impôt des ménages riches diminue alors que celle des ménages pauvres s'accroît (Tableau 17). Le risque de revenu s'exarcerbe et les agents souhaitent davantage épargner pour le motif de précaution. Il s'ensuit une hausse substantielle du patrimoine, s'élevant à $69 \%$ lorsque le taux d'intérêt est invariant à l'excès d'offre de capital. La hausse du patrimoine est en revanche moins conséquente lorsque l'impôt sur les successions devient proportionnel. Elle s'élève à $3,1 \%$ en l'absence de tout mécanisme d'ajustement du taux d'intérêt.

TAB. 17 - Variables agrégées

\begin{tabular}{cccccc}
\hline \hline & & \multicolumn{2}{c}{ Equilibre partiel } & \multicolumn{2}{c}{ Equilibre général } \\
& Référence & IR proportionnel & IS proportionnel & IR proportionnel & IS proportionnel \\
\cline { 2 - 6 }$A$ & 3,51 & 5,93 & 3,62 & 3,80 & 3,52 \\
$T_{I R}^{10 \%, \text { pauvres }}$ & $0 \%$ & $4,7 \%$ & $0 \%$ & $5,3 \%$ & $0 \%$ \\
$T_{I R}^{10 \%, \text {,riches }}$ & $60,5 \%$ & $25 \%$ & $60,2 \%$ & $24,7 \%$ & $60,4 \%$ \\
\hline \hline
\end{tabular}

En équilibre partiel, la hausse de l'indice de Gini est sensiblement plus faible lorsque l'impôt sur le revenu devient proportionnel (tableau 18). De plus, la hausse de l'indice de Gini est plus faible que celle qui découle du passage à la proportionnalité de l'impôt sur les successions en équilibre partiel.

TAB. 18 - Caractéristiques de la distribution du patrimoine en équilibre partiel

\begin{tabular}{cccccc}
\hline \hline & & \multicolumn{2}{c}{ Equilibre partiel } & \multicolumn{2}{c}{ Equilibre général } \\
& Référence & IR proportionnel & IS proportionnel & IR proportionnel & IS proportionnel \\
\cline { 2 - 6 } Indice de Gini & $64,9 \%$ & $66,1 \%$ & $66,3 \%$ & $71,9 \%$ & $66,7 \%$ \\
P75-P100 & $74,3 \%$ & $75,7 \%$ & $75,7 \%$ & $81,5 \%$ & $76,1 \%$ \\
P0-P25 & $0,8 \%$ & $0,63 \%$ & $0,6 \%$ & $0,19 \%$ & $0,56 \%$ \\
P25-P50 & $6,6 \%$ & $5,47 \%$ & $5,7 \%$ & $3,5 \%$ & $5,54 \%$ \\
P0-P50 & $7,4 \%$ & $6,1 \%$ & $6,3 \%$ & $3,7 \%$ & $6,1 \%$ \\
\hline \hline
\end{tabular}


Il apparaît, en outre, que la situation patrimoniale des ménages pauvres (P25-P50) se dégrade beaucoup moins en équilibre partiel lorsque l'impôt sur le revenu devient proportionnel. En équilibre partiel, le taux d'intérêt est plus élevé. Par conséquent, le coût de l'assurance est plus faible.

TAB. 19 - Indice de Gini de la distribution du revenu net de l'impôt en équilibre partiel

\begin{tabular}{lccc}
\hline \hline & \multicolumn{2}{c}{ Equilibre général } & Equilibre partiel \\
& Référence & IR proportionnel & IR proportionnel \\
\cline { 2 - 4 } Indice de Gini & $24 \%$ & $27,6 \%$ & $29,1 \%$ \\
\hline \hline
\end{tabular}

L'accroissement de l'inégalité de revenu consécutive au passage à la proportionnalité de l'impôt sur le revenu est plus conséquent lorsque le taux d'intérêt est invariant à l'excès d'offre de capital. En équilibre général, l'excès d'offre de capital engendre une baisse du taux d'intérêt qui s'accompagne d'une hausse du salaire ${ }^{25}$. Le revenu des ménages riches (resp. pauvres) est principalement composé de revenus du capital (resp. revenus du travail). La baisse du taux d'intérêt réduit davantage les revenus des ménages riches que ceux des ménages pauvres. C'est la raison pour laquelle en équilibre général l'accroissement de l'inégalité de revenu est moindre lorsque l'impôt sur le revenu devient proportionnel.

Ces résultats mettent en exergue l'importance du rôle joué par le taux d'intérêt dans l'accroissement de l'inégalité de patrimoine lorsque l'impôt sur le revenu devient proportionnel.

\subsection{Proportionnalité de l'impôt sur le revenu et seuil d'exemption}

L'analyse menée à la section 4 supposait que le passage à la proportionnalité de l'impôt sur le revenu se faisait sans seuil d'exemption. Autrement dit, aucun ménage n'était exempt de l'impôt sur le revenu. Or, le système d'imposition du revenu est assorti d'un mécanisme de décote qui réduit la facture d'impôt des ménages les plus modestes en termes de revenu. Dans cette section, on se propose de comparer l'impact de la progressivité de l'impôt sur le revenu à celui de la proportionnalité de l'impôt sur le revenu lorsque le mécanisme de décote est pris en compte. La limite de décote est fixée de façon à reproduire le nombre de ménages exempt de l'impôt sur le revenu. Dans ce cas, le taux proportionnel d'imposition qui laisse inchangé le poids de la recette de l'impôt sur le revenu dans le revenu imposable est plus élevé. Il vaut 19,355\%.

En présence d'un mécanisme de décote, la hausse de l'indice de Gini est moindre lorsque l'impôt sur le revenu devient proportionnel (Tableau 21). En raison du mécanisme de décote, $50 \%$ des ménages les plus pauvres continuent d'être exempts de l'impôt sur le revenu. Le montant d'impôt acquitté par les $10 \%$ des ménages les plus riches diminue alors peu. En présence d'un mécanisme de décote, la facture acquittée par les $10 \%$ des ménages les plus riches représente $53,8 \%$ de la facture

\footnotetext{
${ }^{25}$ Celle-ci s'élève à $2,2 \%$.
} 
TAB. 20 - Variables agrégées

\begin{tabular}{ccccc}
\hline \hline & Référence & IR proportionnel & IR Proportionnel+décote & IS proportionnel \\
\hline$A$ & 3,51 & 3,80 & 3,52 & 3,52 \\
$r$ & $3,10 \%$ & $2,4 \%$ & $3,07 \%$ & $3,06 \%$ \\
$T_{I R}^{10 \%, \text { riches }}$ & $60,5 \%$ & $24,7 \%$ & $53,8 \%$ & $60,4 \%$ \\
\hline \hline
\end{tabular}

totale lorsque l'impôt sur le revenu devient proportionnel. En l'absence de mécanisme de décote, celle-ci représente $24,7 \%$ de la facture totale lorsque l'impôt sur le revenu devenait proportionnel (Tableau 20).

TAB. 21 - Caractéristiques de la distribution du patrimoine

\begin{tabular}{ccccc}
\hline \hline & Référence & IR proportionnel & IR proportionnel+décote & IS proportionnel \\
\cline { 2 - 5 } Indice de Gini & $64,9 \%$ & $71,9 \%$ & $67,6 \%$ & $66,7 \%$ \\
P75-P100 & $74,3 \%$ & $81,5 \%$ & $77 \%$ & $76,1 \%$ \\
P0-P25 & $0,8 \%$ & $0,19 \%$ & $0,7 \%$ & $0,56 \%$ \\
P0-P50 & $7,4 \%$ & $3,7 \%$ & $6,7 \%$ & $6,1 \%$ \\
\hline \hline
\end{tabular}

Le risque de revenu s'exacerbe alors moins en présence du mécanisme de décote. Il s'ensuit une hausse de l'épargne de précaution moindre des ménages riches. La hausse du patrimoine est d'ailleurs mineure, inférieure à $1 \%$. La baisse du taux d'intérêt nécessaire à rétablir l'équilibre du marché du capital est moindre. La baisse du taux d'intérêt s'élève à $1 \%$ alors qu'elle est de 22, $6 \%$ en l'absence de mécanisme de décote (Tableau 20). C'est la raison pour laquelle la situation patrimoniale des $25 \%$ des ménages les plus pauvres se dégrade moins lorsque la mise en place de la proportionnalité de l'impôt sur le revenu est assortie d'un mécanisme de décote (Tableau 21). Il en résulte une hausse plus faible de l'indice de Gini.

Cette variante de la proportionnalité de l'impôt sur le revenu conforte l'idée selon laquelle la progressivité de l'impôt sur le revenu a un effet mutualisateur. En raison du mécanisme de décote, l'exacerbation du risque de revenu est moindre suite au passage à la proportionnalité de l'impôt sur le revenu. La réaction des ménages en matière d'épargne de précaution est par conséquent moindre. C'est la raison pour laquelle l'indice de Gini augmente moins.

\section{Conclusion}

Cet article se proposait d'évaluer la contribution de la progressivité de l'impôt sur le revenu et sur les successions à la réduction de l'inégalité de patrimoine en France. Pour ce faire, un modèle à agents hétérogènes qui permet de capturer les inégalités observées de patrimoine, de salaire et de revenu a été construit.

Il est apparu que la progressivité de l'impôt sur le revenu permet de réduire davantage l'inégalité de patrimoine. Le passage à la proportionnalité de l'impôt sur le revenu diminue (resp. augmente) la facture d'impôt des agents riches (resp. pauvres). Le risque de revenu s'en trouve exacerbé. 
Les agents riches épargnent en conséquence davantage pour le motif de précaution. L'inégalité de patrimoine s'accroît alors. En outre, l'accroissement de l'épargne de précaution provoque une baisse du taux d'intérêt qui freine davantage l'accumulation des agents pauvres. Ainsi la baisse du taux d'intérêt participe à l'accroissement de l'inégalité de patrimoine. En revanche, le passage à une imposition proportionnelle des successions exacerbe peu le risque de revenu. Les agents modifient en conséquence peu leur comportement d'épargne de précaution. C'est la raison pour laquelle le passage à une imposition proportionnelle du revenu accroît davantage l'inégalité de patrimoine. Ces résultats suggèrent que la progressivité de l'impôt sur le revenu a probablement joué un rôle important dans la réduction du patrimoine détenu par les agents riches en France, comme le souligne Piketty [2003].

De façon plus attendue, la progressivité de l'impôt sur le revenu s'est avérée très efficace pour réduire l'inégalité de revenu et de consommation car elle a réduit le risque de revenu. En revanche, la progressivité de l'impôt sur les successions semble plus confinée dans le champ du patrimoine.

Si l'approche retenue a le mérite de mettre en exergue les mécanismes par lesquels les progressivités de l'impôt sur le revenu et sur les successions réduisent l'inégalité de patrimoine, un certain nombre de critiques doivent lui être adressées. L'offre de travail est exogène si bien que d'un point de vue de l'efficacité il n'est pas possible d'apprécier les effets (des)incitatifs induits par le caractère proportionnel de l'impôt sur le revenu sur celle-ci et leur impact en retour sur l'inégalité de patrimoine.

Nombre d'économistes s'accordent pour dire que le patrimoine est le résultat de l'accumulation de capital et de transferts intergénérationnels. C'est pourquoi, nous nous sommes attachés à analyser l'impact de la progressivité de l'impôt sur le revenu et sur les successions sur l'inégalité de patrimoine. Néanmoins, il convient de souligner que le système fiscal français s'est vu assorti, depuis 1982, de l'impôt sur les grands fortunes, remplacé en 1989 par l'impôt de solidarité sur la fortune. L'impôt de solidarité sur la fortune est progressif et annuel. Il s'applique à tout patrimoine supérieur à 770000 euros. Les taux marginaux d'imposition varient entre $0,55 \%$ et 1,8\%. Bien que le poids de l'impôt de solidarité sur la fortune dans le PIB est faible, de l'ordre de 0,18\% (Bozio [2007]), il est susceptible de réduire l'inégalité de patrimoine puisque il réduit le patrimoine accumulé chaque année des ménages les plus aisés. Il aurait été intéressant de comparer l'impact de la progressivité de l'impôt de solidarité sur la fortune à celle de l'impôt sur le revenu et de l'impôt sur les successions. 


\section{Annexe}

Le tableau 22 présente les caractéristiques de la distribution du patrimoine des individus en début de retraite (classe d'âge $C_{5}$ ) c'est-à-dire au sortir de la vie active. Les données sont issues de Hairault, Langot et Sopraseulth [2008].

TAB. 22 - Inégalités de patrimoine de la classe d'âge C5

\begin{tabular}{lcc}
\hline \hline & Données & Modèle \\
\cline { 2 - 3 } Patrimoine détenu par P99-P100 & $28 \%$ & $6,9 \%$ \\
Patrimoine détenu par P95-P100 & $49 \%$ & $25,5 \%$ \\
Patrimoine détenu par P80-P100 & $75 \%$ & $61,5 \%$ \\
Patrimoine détenu par P60-P100 & $90 \%$ & $81,9 \%$ \\
Legs détenu par P40-P100 & $97 \%$ & $92,3 \%$ \\
\hline \hline
\end{tabular}

Bien que le modèle parvienne à reproduire de façon satisfaisante la distribution du patrimoine française, la reproduction de la distribution du patrimoine parmi la classe d'âge $C_{5}$ est de qualité moindre au haut de la distribution du patrimoine de cette classe d'âge. Néanmoins, le modèle parvient à approcher la richesse détenue par les $60 \%$ des individus les plus riches de la classe d'âge $C_{5}$. Ce résultat nous conforte dans notre choix d'étalonnage de la matrice $\pi_{x}$ qui décrit les transitions d'une classe d'âge à une autre. 


\section{Références}

[1] Aiyagari, R., 1994, Uninsured idiosyncratic risk and aggregate saving, Quarterly Journal of Economics, 109, 659-84

[2] Algan, Y., Chéron, A., Hairault, J.O., Langot, F., 2003, Wealth effects on labor market transitions, Review of Economic Dynamics, 6, 156-178

[3] Arrondel, L., 2006, Transmission du patrimoine et impôt successoral. L'impact sur les transferts entre générations, Informations sociales, 134, 42-53

[4] Arrondel, L., Laferrère, A., 1991, Successions et héritiers à travers les données fiscales, Economie et Prévision, 100-101, 137-175

[5] Arrondel, L., Laferrère, A., 2001, Taxation and wealth transmission in France, Journal of Public Economics, 79, 3-33

[6] Asdrubali, P., Sørensen, B.E., Yosha, O., 1996, Channels of interstate risk sharing : United States 1963-1990, Quarterly Journal of Economics, 104, 1081-1110

[7] Bourguignon, F., Bureau, D., 1999, L'architecture des prélèvements en France : état des lieux et voies de réforme, rapport du conseil d'Analyse Economique, $\mathrm{n}^{\circ} 17$, la documentation française

[8] Bozio, A., 2007, La taxation du patrimoine en France. Enjeux, bilan et perspectives, Regards croisés sur l'économie, $\mathrm{n}^{\circ} 1,204-210$

[9] Cagetti, M., De Nardi, M., 2006, Entrepreneurship, frictions, and wealth, Journal of political Economy, 114, 835-870

[10] Castaneda, A., Diaz-Gimenez, J., Rios-Rull, J.V., 1998, Earnings and wealth inequality and income taxation : quantifying the trade-offs of switching the U.S. to a proportional income tax system, Technical Report, PEN University

[11] Castaneda, A., Diaz-gimenez, J., Rios-Rull, J.V., 2003, Accounting for the U.S. earnings and wealth inequality, Journal of Political Economy, 111, 818-857

[12] Chambaz, C., Guillaumat-Tailliet, F., Hourriez, J.M., 1999, Le revenu et le patrimoine des ménages, Données sociales, INSEE

[13] Charpin, J.M., 1999, L'avenir de nos retraites, La documentation francaise

[14] Conesa, J.C., Krueger, D., 2006, On the optimal progressivity of the income tax code, Journal of Monetary Economics, 53, 1425-1450

[15] Colin, C., 1999, Modélisation des carrières salariales dans DESTINIE, Document de travail $n^{\circ}$ G9902 de la Direction des études et synthèses économiques, Division redistribution et politiques sociales

[16] Davies, J., 1982, The relative impact oh inheritance and other factors on economic inequality, Quarterly Journal of Economics, 97, 471-498 
[17] De Nardi, M., 2004, Wealth inequality and intergenerational links, Review of Economic Studies, $71,743-768$

[18] Direr, A., Weitzenblum, T., 2006, Modéliser la distribution des richesses en France, Annales d'Economie et de Statistiques, 82, 151-186

[19] Domeij, D., Heathcote, J., 2004, On the distributional effects of reducing capital taxes, International Economic Review, 45, 523-554

[20] Ducamin, B., Baconnier, R., Briet, R., 1996, Etudes des prélèvements fiscaux et sociaux pesant sur les ménages : rapport au ministre du budget, Ministère du Budget

[21] Eaton, J., Rosen H., 1980, Taxation, human capital, and uncertainty, American Economic review, 70, 705-715

[22] Gokhale, J., Kotlikoff, L., Sefton, J., Weale, M., 2001, Simulating the transmission of wealth inequality via bequests, Journal of Public economics, 79, 93-128

[23] Gouveia, M., Strauss, R.P. (1994). Effective federal individual income tax functions : an exploratory empirical analysis, National Tax Journal, 47, 317-339

[24] Grant, C., Koulovatianos, C., Michaelides, A., Padula, M., 2003, Redistributive policies through taxation : theory and evidence, CSEF Working Paper, University of Salerno

[25] Hairault, J.O, Langot, F., 2002, Inégalités et réformes des retraites, Revue Française d'Economie, $1,97-161$

[26] Hairault, J.O, Langot, F., 2008, Inequality and social security reforms, Journal of Economic Dynamics and Control, 32, 386-410

[27] Hairault, J.O., Langot, F., Sopraseulth, T., 2008, Quantifying the Laffer curve on the continued activity tax in a dynastic framework, International Economic Review, 49, 755-797

[28] Hourriez, J.M., Roux, V., 2001, Vue d'ensemble des inégalités, Document de travail INSEE F0103

[29] Kessler, D., Wolff, E., 1991, A comparative analysis of household wealth patterns in France and the United States, Review of Income and Wealth, 37, 249-266

[30] Kotlikoff, L., Summers, L., 1981, The role of intergenerationnal transfers in aggregate capital accumulation, Journal of Political Economy, 89, 706-732

[31] Kotlikoff, L., Summers, L., 1988, The contribution of intergenerational transfers to total wealth : a reply, Modelling the accumulation and distribution of wealth, Clarendon Press, $53-67$

[32] Laitner, J., 2001, Wealth accumulation in the US : do inheritances and bequests play a significant role?, Working Paper, University of Michigan

[33] Lefranc, A. Trannoy, A, 2005, Intergenerational earnings mobility in France? Is France more mobile than the US, Annales d'Economie et Statistique, 78, 57-78 
[34] Lollivier, S., Verger, D., 1996, Patrimoine des ménages : déterminants et disparités, Economie et Statistique, 296-297, 13-31

[35] Modigliani, F., 1988a, Measuring the contribution of intergenerational transfers to total wealth : conceptual issues and empirical findings", Modelling the accumulation and distribution of wealth, Clarendon Press, 21-52

[36] Modigliani, F., 1988b, The Role of intergenerational transfers and life cycle saving in the accumulation of wealth, Journal of Economic Perspectives, 2, 15-40

[37] Piketty, T., 1997, L’Economie des inégalités, La découverte

[38] Piketty, T., 2001, Les hauts revenus en France au XXe siècle. Inégalités et redistributions,19011998, Grasset

[39] Piketty, T., 2003, Income inequality in France, 1901-1998, Journal of Political Economy, 111, 1004-1042

[40] Quadrini, V., 2000, Entrepreneurship saving and social mobility, Review of Economic Dynamics, 3, 1-40

[41] Rougerie, C., 2002, Evolution des inégalités de patrimoine chez les salariés entre 1986 et 2000, Données sociales, INSEE

[42] Tauchen, G., Hussey, R., 1991, Quadrature-based methods for obtaining approximate solutions to nonlinear asset pricing models, Econometrica, 59, 371-396

[43] Varian, H.R., 1980, Redistributive taxation as social insurance, Journal of Public Economics, $14,49-68$

[44] Ventura, G., 1999, Flat Tax Reform : A Quantitative exploration, Journal of Economic Dynamics and Control, 23, 1425-1458 